\title{
Reliably predicting FIR lines from simulated galaxies
}

\author{
Alessandro Lupi, ${ }^{1 \star}$ Andrea Pallottini, ${ }^{1}$ Andrea Ferrara, ${ }^{1}$ Stefano Bovino, ${ }^{2}$ \\ Stefano Carniani ${ }^{1}$, and Livia Vallini ${ }^{3}$ \\ ${ }^{1}$ Scuola Normale Superiore, Piazza dei Cavalieri 7, Pisa IT-56126, Italy \\ ${ }^{2}$ Departamento de Astronomía, Facultad Ciencias Físicas y Matemáticas, Universidad de Concepción, \\ Av. Esteban Iturra s/n Barrio Universitario, Casilla 160, Concepción, Chile \\ ${ }^{3}$ Leiden Observatory, Leiden University, PO Box 9500, 2300 RA Leiden, The Netherlands
}

Accepted XXX. Received YYY; in original form 15 April 2020

\begin{abstract}
Far-infrared (FIR) emission lines are a powerful tool to investigate the properties of the interstellar medium, especially in high-redshift galaxies, where ALMA observations have provided unprecedented information. Interpreting such data with state-of-the-art cosmological simulations post-processed with CLOUDY, has provided insights on the internal structure and gas dynamics of these systems. However, no detailed investigation of the consistency and uncertainties of this kind of analysis has been performed to date. Here, we compare different approaches to estimate FIR line emission from state-of-theart cosmological simulations, either with CLOUDY or with on-the-fly non-equilibrium chemistry. We find that $[\mathrm{CII}]_{158 \mu}$ predictions are robust. [OI] emission lines are instead model-dependent, as these lines are strongly affected by the thermodynamic state of the gas and non-equilibrium photoionisation effects. For the same reasons, [OI] lines represent an excellent ISM diagnostic. Future observations targeting these lines will be also crucial to constrain models.
\end{abstract}

Key words: galaxies: formation, galaxies: evolution, galaxies: high-redshift, galaxies: ISM, ISM: kinematics and dynamics, ISM: lines and bands

\section{INTRODUCTION}

In the last decades, deep high-resolution observations in the optical/near-infrared band have allowed us to start probing the high-redshift Universe and the Epoch of Reionisation, providing important insights on galaxy formation and evolution processes. However, to get a proper characterisation of the first galaxies, in particular the properties of the interstellar medium (ISM) at these redshifts, detailed spectral information is necessary. Low redshift observations have shown that fine structure lines in the far-infrared band like $[\mathrm{CII}]_{158 \mu},[\mathrm{OI}]_{63 \mu}$, and $[\mathrm{OIII}]_{88 \mu}$ - represent good tracers of the interstellar medium (ISM) thermodynamic state, and of the star formation process (De Looze et al. 2014; Herrera-Camus et al. 2015). These lines are typically unaffected by dust, unlike the ultraviolet (UV) emission, and can be equally bright in the high-redshift Universe (e.g. Carniani et al. 2018; Hashimoto et al. 2019). At high-redshift, FIR lines are shifted to the sub-mm band, where are accessible by ALMA. In the last few years, ALMA observations have given us unprecedented data at extremely high resolution,

^ E-mail: alessandro.lupi@sns.it providing important information about the ISM properties and kinematics of high-redshift sources.

Theoretically, several models have been developed to investigate the properties of high-redshift systems. While many of them focused on the relative impact of stellar feedback (e.g. Pallottini et al. 2017a; Trebitsch et al. 2018; Ma et al. 2018; Katz et al. 2017, 2019), the chemical composition (Maio \& Tescari 2015; Arata et al. 2020), and the role of active galactic nuclei (Trebitsch et al. 2019; Lupi et al. 2019), other studies tried to more accurately describe how the FIR emission is affected by different gas conditions, (e.g. Vallini et al. 2013; Olsen et al. 2015), also considering the impact of the cosmic microwave background and the metallicity on the powered emission (Vallini et al. 2015; Olsen et al. 2017), internal structure of molecular clouds (Vallini et al. 2018; Decataldo et al. in prep), the importance of the radiation field (Vallini et al. 2017; Pallottini et al. 2019; Arata et al. 2019), and possible bias in the comparison between theory and simulations (Kohandel et al. 2019; Lupi et al. 2019).

In the majority of these studies, emission lines are estimated via post-processing with CLOUDY (Ferland et al. 1998), that provides the most accurate treatment to date of atomic and molecular microphysics, accounting for impinging radiation field and different ISM conditions. However, 
because of its complexity and computational cost, applying CLOUDY calculations on-the-fly in hydrodynamic simulations is not currently feasible, and cheaper approximate approaches have been developed, as machine learning techniques (Katz et al. 2019) or multi-dimensional interpolation of tabulated emission lines (Pallottini et al. 2019). Moreover, CLOUDY assumes photo-ionisation equilibrium conditions, which does not always give a good representation of the real thermodynamic state of the ISM, especially for warm and cold gas (Bovino et al. 2016), and can produce less accurate heating/cooling rates for the gas in galaxies (Richings et al. 2014; Richings \& Schaye 2016).

An alternative way to model emission lines is the direct inclusion of non-equilibrium calculations within simulations, providing all the necessary chemical abundances at every time. The abundances and thermodynamic state of the gas can then be used to compute the emission lines by estimating the excitation level population of each ion assuming a statistical equilibrium among the levels. This approach ensures a better representation of the evolution of the ISM, thanks to the consistent coupling of chemistry and thermodynamics. The time-dependent species abundances and cooling/heating processes thus ensure a strong interplay with all the sub-grid physics models included in the simulation. However, because of its complexity, this approach is currently feasible only for simplified chemical networks with a limited number of species (e.g. Capelo et al. 2018; Lupi \& Bovino 2020).

A crucial role in the emission line modelling is the temperature considered for each cell, that can be directly taken from the simulation, hence without considering any subresolution structure, as typically done in KROME and in Katz et al. (2019), or allowing for a depth-dependent distribution computed according to photo-ionisation equilibrium Pallottini et al. (2019).

In this study, we address how robust the FIR emission line prediction obtained in simulations is, depending on the photo-ionisation equilibrium and thermal state assumptions for the gas, by comparing post-processing CLOUDY calculations and on-the-fly non-equilibrium chemistry. To achieve this goal, we employ a state-of-the-art high-resolution zoomin cosmological simulation of a typical star forming galaxy at $z=6$; the simulation includes a non-equilibrium chemical network, a physically-motivated star formation model, and stellar feedback by supernovae, winds, and radiation, the latter evolved through on-the-fly radiative transfer calculations. The paper is organised as follows: in Section 2 we describe the numerical setup and the sub-grid models employed; in Section 3 we present our simulation results, in Section 4 we discuss the emission line properties, and in Section 6 we draw our conclusions.

\section{NUMERICAL SETUP}

We perform a cosmological zoom-in simulation targeting a $M_{\mathrm{vir}} \sim 3 \times 10^{11} \mathrm{M}_{\odot}$ halo at $z=6$ using the hydrodynamic code GIZMO (Hopkins 2015), descendant of GADGET3 and GADGET2 (Springel 2005), employing the meshless-finitemass method. Gravity is solved via a TreePM approach, with the maximum spatial resolution set by the Plummerequivalent gravitational softening (kept fixed across the run) at $30 \mathrm{pc}$ for dark matter (DM) and $10 \mathrm{pc}$ for stars. For gas particles/cells, we employ adaptive softenings, with a minimum softening of $3 \mathrm{pc}$. The mass resolution is $\sim 10^{5} \mathrm{M}_{\odot}$ for $\mathrm{DM}$ and $2 \times 10^{4} \mathrm{M}_{\odot}$ for gas and stars. The initial conditions are created with MUSIC (Hahn \& Abel 2013) assuming the Planck Collaboration et al. (2016) cosmological parameters, where $\Omega_{\mathrm{m}}=0.308, \Omega_{\mathrm{b}}=0.0481, \Omega_{\Lambda}=0.692$, $H_{0}=67.74 \mathrm{~km} \mathrm{~s}^{-1} \mathrm{Mpc}^{-1}, \sigma_{8}=0.826$, and $n_{\mathrm{s}}=0.9629$.

The simulation presented here is performed employing the sub-grid model described in Lupi \& Bovino (2020), with the addition of the M1 on-the-fly radiation transport (Levermore 1984; Hopkins et al. 2020).

\section{$2.1 \quad$ Sub-grid modelling}

Here, we recall the sub-grid models employed in our run, and describe the coupling between chemistry and radiation.

- Radiative cooling and chemistry: we employ the nonequilibrium chemistry library KROME (Grassi et al. 2014). Our network follows sixteen chemical species, i.e. $\mathrm{H}, \mathrm{H}^{-}$, $\mathrm{H}^{+}, \mathrm{H}_{2}, \mathrm{H}_{2}^{+}, \mathrm{He}, \mathrm{He}^{+}, \mathrm{He}^{++}, \mathrm{C}, \mathrm{C}^{+}, \mathrm{O}, \mathrm{O}^{+}, \mathrm{Si}, \mathrm{Si}^{+}$, and $\mathrm{Si}^{++}$, properly taking into account the metal contribution to gas cooling for $T<10^{4} \mathrm{~K}$ (instead of equilibrium tables), and the ultra-violet background (UVB) by Haardt \& Madau (2012), as in Capelo et al. (2018) and Lupi \& Bovino (2020). Photo-chemistry is implemented accounting for the photon flux within each cell in ten different radiative bins spanning the range 0.75-1000 eV (Lupi et al. 2018).

- Star formation (SF): SF is modelled via a stochastic approach with the efficiency $\varepsilon_{\mathrm{SF}}$ computed on a cell/particle basis as a function of local gas properties (density, thermal and turbulent support). This prescription, already employed in Lupi \& Bovino (2020) and based on the theoretical model by Padoan et al. (2012), assumes that every particle/cell in the simulation represents a entire molecular cloud (or a large portion of it) described by a Log-Normal probability distribution function, whose parameters (Mach number, mean density, and virial parameter) are determined on-the-fly according to the local gas properties (Lupi et al. 2019; Lupi \& Bovino 2020). As in Semenov et al. (2017), the local efficiency within the cloud is assumed to be 90 per cent at the pre-stellar core scale.

- Stellar winds and supernova feedback: due to the limited mass resolution, every stellar particle is assumed to represent an entire population following a Kroupa (2001) Initial Mass Function (IMF). Energy, mass, and metals are released by SNe in discrete events (Hopkins et al. 2018; Lupi 2019) of $10^{51} \mathrm{erg}$ each, where thermal energy and momentum are directly added to the gas according to the results by Martizzi et al. (2015). As in our previous studies (Lupi et al. 2018; Lupi 2019; Lupi et al. 2019), metal injection by SNe is computed by scaling $\mathrm{O}$ and $\mathrm{Fe}$ abundances to a total $\mathrm{Z}$ according to the solar ratios (Asplund et al. 2009), that give $M_{Z}=2.09 M_{\mathrm{O}}+1.06 M_{\mathrm{Fe}}$ (Kim et al. 2014). For stellar winds, we do not consider any additional metal production relative to the stellar progenitor metal fraction, and dump feedback as thermal energy only, in addition to the initial ejecta momentum. ${ }^{1}$

${ }^{1}$ In order to properly conserve species abundances for the chemical evolution, metal species (and ions) abundances are re-scaled, 
- Stellar radiation: We model stellar radiation assuming the up-to-date Bruzual \& Charlot (2003) stellar population synthesis models for a Kroupa IMF. The spectra are sampled in ten radiation bins as a function of stellar age and metallicity, with each stellar particle representing an entire stellar population.

\subsection{Radiation transport and photo-chemistry}

In our simulation, we employ a moment-based radiation transport (RT) scheme based on the widely used M1 closure scheme (Rosdahl et al. 2013; Hopkins et al. 2020), where the first and second momenta of the RT equation are solved assuming a purely local form of the Eddington tensor (Levermore 1984). This scheme results in hyperbolic equations, that can be solved with the Godunov-like method also employed for hydrodynamics (Aubert \& Teyssier 2008). To make the run feasible, we employ the reduced speed of light approximation (Gnedin \& Abel 2001) with a reduction factor $f_{c}=0.001$.

In its standard implementation within GIZMO, the signal speed eigenvalues are derived under the HLL approximation, and are obtained from a fit to the two-dimensional table created by Rosdahl et al. (2013). Here, we opt instead for the more accurate (and purely analytical) derivation reported in Skinner \& Ostriker (2013), that gives

$\frac{\lambda_{1,3}}{\tilde{c}}=\left\{\mu f \pm\left[\frac{2}{3}\left(\xi^{2}-\xi\right)+2 \mu^{2}\left(2-f^{2}-\xi\right)\right]^{1 / 2}\right\} / \xi$,

where $\tilde{c}=f_{c} c, c$ is the speed of light, $f=\left|F_{v}\right| /\left(\tilde{c} E_{v}\right)$ is the reduced flux, $\mu=\cos \theta$ is the angle between the radiation flux and the face vectors, and $\xi=\sqrt{4-3 f^{2}}$.

At every time-step, all active stellar particles inject photons into the nearest $N_{\text {ngbs }} \approx 64$ gas neighbours ${ }^{2}$, weighted by a parabolic kernel as a function of distance, as it is done in the public version of GIZMO. Because of the intrinsic particlebased nature of GIZMO, radiation cannot be injected in a single 'host cell' as commonly done in grid-based codes, but is spread over a discrete number of neighbours. This process could in principle artificially boost the escape of radiation from high-density regions, and overestimate the radiative feedback of the star, especially if the farthest neighbours are optically thin to radiation. In order to prevent this issue, and account for unresolved absorption, for every energy bin, we attenuate the photons injected accounting for the column density between the source and the target cell $j$ as

$\Delta N_{\gamma, j}^{\prime}=\Delta N_{\gamma, j} \exp \left(-\kappa_{j} \rho_{j} r_{\mathrm{eff}, j}\right)$,

where $\kappa_{j}$ and $\rho_{j}$ are the neighbour cell opacity and density and $r_{\mathrm{eff}, j}=\max \left\{l_{\text {host }}, r_{\star, j}\right\}$, with $l_{\text {host }}$ is the size of the 'virtual host cell' around the stellar source, and $r_{\star, j}$ is the separation between the target cell and the source. As in Hopkins et al. (2018) and Lupi (2019), we define $l_{\text {host }}=\left[3 H_{\text {host }} /\left(4 \pi N_{\mathrm{Ngb}}\right)\right]^{1 / 3}$, where $H_{\text {host }}$ is the kernel size of the star encompassing $N_{\mathrm{Ngb}}=64$ neighbours. A schematic

for active gas particles, to match the updated total metallicity of the particle.

2 The number of neighbours for stars is twice that of the gas, and this choice is made to ensure that the region around the source is well sampled (see, e.g., Lupi 2019, for details)

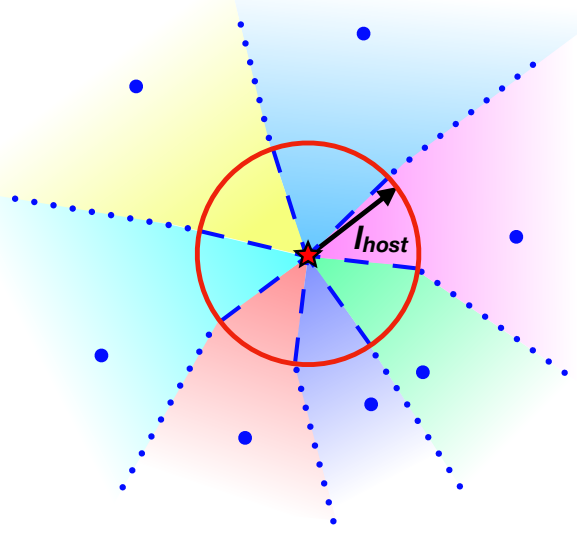

Figure 1. Schematic view of the sub-grid absorption applied during the photon injection step, to account for unresolved absorption in the simulation and prevent the artificial excess of escaping radiation. The red circle represents the 'virtual host cell' around the source, with size $l_{\text {host }}$; the different colours correspond to the region associated to each neighbouring gas particle $j$, that determines the fraction of the total luminosity dumped on that particle itself.

view of this approach is reported in Fig. 1. In addition to this correction, we also account for the unresolved radiation pressure on the neighbours, by imparting a kick defined $a^{3}$

$m_{j} \Delta \mathbf{v}=\frac{\Delta E_{\gamma, j}}{c}\left[1-\exp \left(-k_{j} \rho_{j} r_{\mathrm{eff}, j}\right)\right]$,

where $\Delta E_{\gamma, j}=\Delta N_{\gamma, j}\left\langle E_{\gamma}\right\rangle$ is the photon energy injected and $\left\langle E_{\gamma}\right\rangle$ the average photon energy per bin.

To couple radiation with chemistry, we follow the same approach described in Lupi et al. (2018), where photo-rates and photo-heating are computed in KROME from the photon fluxes within each cell of the simulation, defined for every radiation bin $j$ as $F_{\gamma, j}=\tilde{c} E_{\gamma, j} / V_{\text {cell }}$, with $E_{\gamma, j}$ the $j$-th bin photon energy, and $V_{\text {cell }}=h_{\text {cell }}^{3}$ the cell volume. While the opacity resulting from the abundance of the species included in the chemical network is consistently treated by KROME, and used to properly attenuate radiation in the RT scheme, dust shielding and $\mathrm{H}_{2}$ self-shielding are included only in the chemistry calculations assuming an effective absorption scale equivalent to the Jeans length capped at $40 \mathrm{~K}$ (SafranekShrader et al. 2017) for each gas cell/particle (Lupi et al. 2019).

\section{RESULTS}

We now present our results, and discuss the uncertainties in the predicted emission lines for our target galaxy. For all the analyses reported here, we have identified the

3 An unresolved radiation pressure term is already implemented in the public version of GIZMO, but uses a different definition for the absorption length, i.e. $r_{\text {eff, } j}=2 \max \left\{2 h_{j}, H_{\text {host }}\right\}$, with $h_{j}$ the effective size of the $j$-th cell. 
$M_{\text {vir }} \sim 3 \times 10^{11} \mathrm{M}_{\odot}$ target halo using AMIGA HALO FINDER (Knollmann \& Knebe 2009), and we have considered all the particles/cells within 20 per cent of the halo virial radius, in order to exclude any contamination by satellites. As an example, at $z \sim 6$, the halo virial radius is about $28.4 \mathrm{kpc}$, hence we consider particles within $5.7 \mathrm{kpc}$ only.

\subsection{Galaxy evolution across cosmic time}

In the top-left panel of Fig. 2, we show the redshift evolution of the mass for different components. The stellar mass build-up starts about 200 Myr after the Big Bang, but at a moderately slow pace until $z \sim 12$, when the halo exceeds $M_{\text {halo }} \sim 10^{10} \mathrm{M}_{\odot}$ and SNe stop evacuating most of the gas. This is reflected in the initially bursty SFR shown in the topright panel, that becomes steadier and converges to about $40-50 \mathrm{M}_{\odot} \mathrm{yr}^{-1}$ below $z \sim 10-9$. At very high redshift, gas makes up for most of the baryons, with neutral hydrogen representing the most abundant element, and molecular hydrogen making up for less than $5 \%$. Below $z=9$, stars start to dominate, and the gas-to-star ratio settles around 30 per cent (corresponding to a gas fraction $f_{g}=0.23$ ), with neutral hydrogen contributing for about $40-50$ per cent, and the molecular component up to 15 per cent (see, also, Popping et al. 2015). While at $z \gtrsim 9$ our galaxy is consistent with the empirical stellar-to-halo mass relation by Behroozi et al. (2013) and Behroozi et al. (2019), at $z \sim 6$ our stellar mass $\left(M_{\text {star }}=1.6 \times 10^{10} \mathrm{M}_{\odot}\right)$ is about $2-2.5$ times above the relation, because of the weaker effect of $\mathrm{SNe}$ at larger halo masses, although still compatible within $3 \sigma$.

The SFR, instead, is roughly constant around $50 \mathrm{M}_{\odot} \mathrm{yr}^{-1}$ below $z \sim 8$, in perfect agreement with the observational constraints by Salmon et al. (2015), for which $\dot{M}_{\text {star }} \approx 45 \mathrm{M}_{\odot} \mathrm{yr}^{-1}$, and the empirical estimates by Behroozi et al. (2013), for which $\dot{M}_{\text {star }} \approx 72 \mathrm{M}_{\odot} \mathrm{yr}^{-1}$.

In the bottom-left panel, we also show the stellar and gas metallicity of the galaxy. While the stellar metallicity increases almost monotonically with time, reaching solar values around $z=7.5$, gas exhibits stronger fluctuations, due to the competing effect of SN enrichment and pristine gas inflows. Around $z=7-6$, the average gas metallicity has saturated around $Z_{\text {gas }}=0.5 \mathrm{Z}_{\odot}$, whereas stars are twice more metal-rich relative to gas. The gas metallicity we get is consistent with the estimates for high-redshift galaxies observations (e.g. Carniani et al. 2018) and similar simulations (e.g. Pallottini et al. 2017b).

Finally, in the bottom-right panel, we report the size evolution of the different galaxy components. The half-mass radius for the stellar component is shown as a red solid line, $\mathrm{H} \mathrm{I}$ as green dashed one, and $\mathrm{H}_{2}$ as a cyan dotted one. At early times, only a few clumps exist, where $\mathrm{H}_{2}$ is embedded within neutral gas. This results in a comparable size for the two components, whereas stars only form in the densest region at the centre of the system, resulting in a more concentrated distribution. At $z \lesssim 11$, the occurrence of a major merger results in an apparent rapid size increase, with the peak at $\sim 800$ pc at $z \sim 10$, followed by a sharp drop in size when the merger ends and the galaxy remnant settles $(z \sim 8-9)$. Below $z=8$, the galaxy exhibits a well-defined gaseous disc, mostly $\mathrm{H}_{2}$ rich, where stars continuously form, whereas the remaining neutral gas is more extended, up to a few $\mathrm{kpc}$. Around $z=6$, the stellar distribution remains quite compact, with a typical half-mass (and half-light) radius of about 300-400 pc, a value only moderately smaller than the typically observed one for Lyman-break galaxies (see, e.g., Figure 32 in Dayal \& Ferrara 2018).

\subsection{Galaxy properties at $z=6$}

Next, we focus on the properties of the galaxy at $z \sim 6$, with particular emphasis on the ISM. In Fig. 3, we show the spatial distribution of the different galaxy components. The stellar distribution (top-left panel) exhibits a smooth disc structure, with well defined spiral arms. The galaxy is compact, with most of the stellar mass within $1 \mathrm{kpc}$ (the total half-mass radius is about $300 \mathrm{pc}$, see bottom-right panel of Fig. 2). Using the intrinsic FUV emission, computed from the Bruzual \& Charlot (2003) stellar spectra in the band $1350-1750 \AA$, we can identify star forming regions (bottomleft panel): young stars forming from the gaseous disc also settle on a disc, although the distribution is more clumpy, reflecting the clustering of stars forming within molecular clouds. With time, stellar radiation, winds, and $\mathrm{SNe}$ evacuate gas from the clouds, and the stellar clusters slowly disperse within the disc.

Compared to local galaxies, high-redshift systems continuously accrete gas from the environment, and this provides new fuel to sustain SF (which in these systems can easily exceed $10 \mathrm{M}_{\odot} \mathrm{yr}^{-1}$ ). As shown by the total gas column density (first row, central panel), a significant amount of gas extends up to several kpc; such gas has either been expelled by stellar feedback or is flowing in from large-scale filaments. On the contrary, main galaxy disc does not exceed 1-2 kpc. The $\mathrm{H}_{2}$ distribution (central panel, second row) closely follows that of young stars, consistently with expectations that SF mostly occurs in cold and dense molecular clouds where $\mathrm{H}_{2}$ is abundant. We stress that the $\mathrm{H}_{2}$ distribution in our run is a natural byproduct of our simulation, despite we do not assume any dependence of SF on the $\mathrm{H}_{2}$ abundance (Lupi et al. 2018). Finally, in the right panels, we report the average gas temperature along the line-of-sight (top panel), and $\mathrm{H}$ ionization fraction, $x_{\mathrm{H}^{+}}$(bottom panel). Most of the gas in the spiral arms is cold, as expected for $\mathrm{H}_{2}$-dominated gas, whereas the inter-spiral regions with lower density gas are warm/hot, as they are heated and ionized by stellar radiation. Outside the galactic disc, the gas is typically hotter, with warm filaments embedded within low-density regions with high temperatures, as also highlighted by the ionisation fraction that approaches unity.

One of the most fundamental correlations between galaxy properties is the Schmidt-Kennicutt (KS) relation (Schmidt 1959; Kennicutt 1998), that links the neutral and molecular gas reservoir to the effective SFR. In Fig. 4, we show the KS relation in total gas from observations of different systems (normal local spirals as well as highredshift or starburst galaxies) with our run. We show the best-fit by Kennicutt (1998) (orange shaded area and solid line), the spatially-resolved local measures by Bigiel et al. (2010) (filled red/green contours), and the measures of starburst (SB), sub-millimeter (SMG), and ultra-luminous infrared (ULIRGs) galaxies reported in Daddi et al. (2010) (gray squares). In our run (where the red points correspond to $0.3 \mathrm{kpc}$ patches), the low-density region matches the local data very well, also exhibiting the kink around 

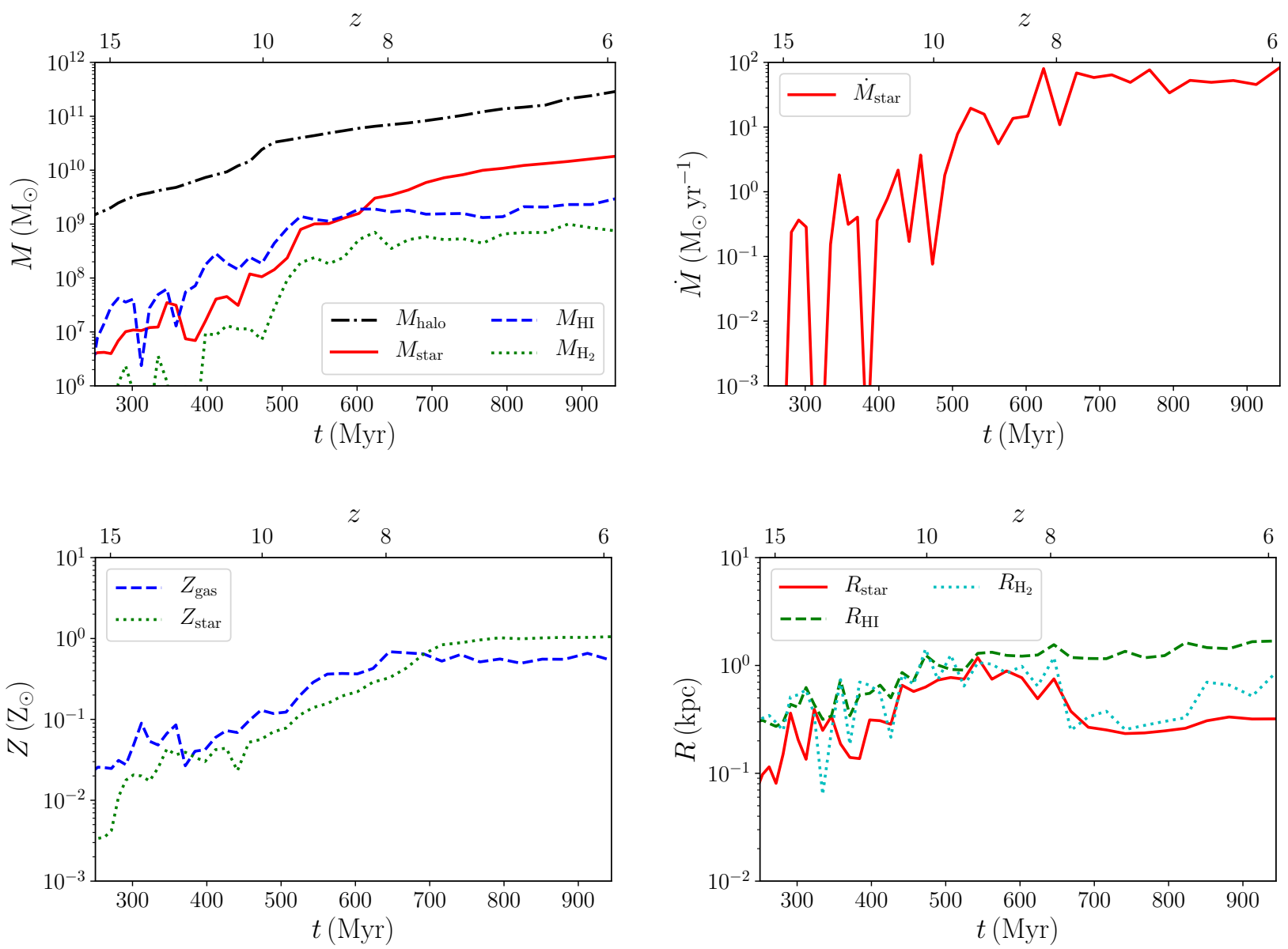

Figure 2. Redshift evolution of galaxy properties. Top-left panel: halo mass (black dot-dashed line), stellar mass (red solid), the neutral hydrogen mass (blue dashed line), and the molecular hydrogen mass (green dotted) Top-right: Star formation rate (SFR, red solid line). Bottom-left: gas (blue dashed) and stellar (green dotted) metallicity. Bottom-right: Half-mass radii for stars (red solid line), HI (green dashed), and $\mathrm{H}_{2}$ (cyan dotted).

$\Sigma_{\mathrm{H}+\mathrm{H}_{2}}=10 \mathrm{M}_{\odot} \mathrm{yr}^{-1}$, whereas the higher density patches settle in the starburst regime Daddi et al. (2010). For completeness, we also show the average values for our galaxy as star symbols. The empty one corresponds to the average obtained at $2 \mathrm{kpc}$ resolution, whereas the filled one is obtained as the ratio between the total $\mathrm{H}+\mathrm{H}_{2}$ mass (SFR) within $4 \mathrm{kpc}$ and twice the surface of the region encompassing half of the $\mathrm{H}_{2}$ (stars) mass, similarly to Miettinen et al. (2017). ${ }^{4}$ In both cases, our galaxy matches the starburst data by Daddi et al. (2010), further confirming the results obtained with the patches. This suggests that, although high- $z$ galaxies are more likely to exhibit star-bursting phases, with SFRs well above the local relation, the spatially-resolved relation is not dissimilar from the local one, with lower density regions $\left(\Sigma_{\mathrm{H}+\mathrm{H}_{2}} \lesssim 40 \mathrm{M}_{\odot} \mathrm{pc}^{-2}\right)$ perfectly consistent with nearby disc galaxies, and the densest regions within the disc $\left(\Sigma_{\mathrm{H}+\mathrm{H}_{2}} \gtrsim 40 \mathrm{M}_{\odot} \mathrm{pc}^{-2}\right)$ reproducing low-redshift starbursts.

${ }^{4}$ We note that, if the considered radii are significantly different, this choice can artificially bias the correlation, hence the use of similar radii to average both quantities, as usually done when the galaxy is resolved, is preferred.
Since most of the emission associated with newly formed stars comes from the densest regions, these early galaxies are likely to appear as starbursts, as found by Vallini et al. (2020). However, because of the limited resolution and sensitivity of current observations, such an analysis cannot be performed on many sources, and a clear consensus about their location relative to the KS relation is still missing (see, e.g. Pavesi et al. 2019).

\section{TESTING FIR LINE PREDICTIONS}

Now, we focus on the FIR emission from the galaxy at $z=6$, and investigate how the assumptions on the thermodynamic and ionisation state of the gas affect the estimated flux. This is crucial to assess the robustness of predictions, and how observations could help constraining the sub-grid prescriptions adopted in simulations (Olsen et al. 2018).

\subsection{Modelling emission lines}

FIR emission lines can be inferred from numerical simulations following different approaches. In particular, in this 


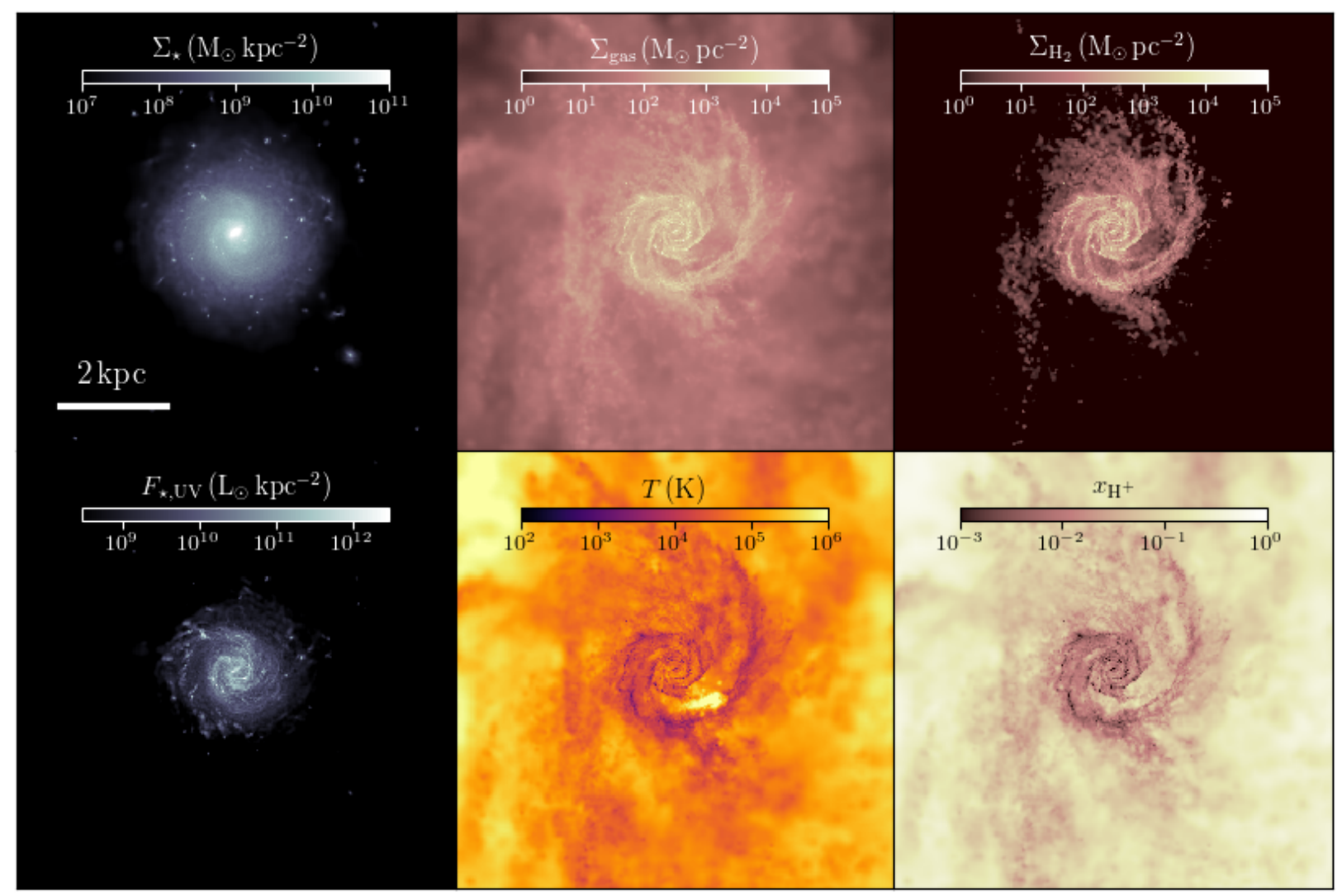

Figure 3. Maps of the key properties of our simulated galaxy at $z \sim 6$. The top-left panel shows the stellar surface density, whereas the intrinsic FUV flux from the stellar spectra, tracing young stars, is shown in the bottom-left panel. In the second column, the total gas column density is reported in the top one, and the $\mathrm{H}_{2}$ column density in the bottom one. Finally, in the top-right panel, we report the line-of-sight averaged temperature of the galaxy, and in the bottom-right one the ionised hydrogen fraction $x_{\mathrm{H}^{+}}$. By comparing the FUV emission with $\mathrm{H}_{2}$, we notice that $\mathrm{H}_{2}$ traces well the distribution of young stars forming within molecular clouds, whereas the global stellar distribution in the galaxy (leftmost panel), still dominated by older stars, is more concentrated. Compared to the molecular component, the neutral and ionised components are more diffuse, extending up to several kpc, as highlighted by the temperature and the $x_{\mathrm{H}^{+}}$maps.

Table 1. Differences between CLOUdy and KROME in the method emission lines are estimated and in the assumptions made for the chemical/thermodynamic state of the gas. All the features reported here refer to single cell calculation at a specific time, and do not reflect how thermodynamics is evolved during the simulation. In particular, CloudyFIX and CloudyVAR differ in how the temperature is evolved within the slab, either constant and based on the simulation outputs, in this way taking into account any dynamical effect (CloudyFIX), or let free to vary according to the radiation attenuation computed by CLOUDY (CloudyVAR).

\begin{tabular}{llll}
\hline \hline Feature & Krome & CloudyVAR & CloudyFIX $^{\dagger}$ \\
\hline Spatial structure & Average properties (0D) & slab (1D) & \\
Minimum size considered & as simulation resolution & optically thin slices & Partial \\
Coupling with the simulation feedback & Self-consistent & No & \\
Chemical network & 5 atoms and 1 molecule & 30 atoms and multiple molecules & \\
Atomic levels hierarchy & Dominant levels only & Full & Constant T \\
Chemical state & Full non-equilibrium & Ionisation equilibrium & Yes \\
Thermodynamic state & Constant T & Variable T & No \\
Effect of shocks & Yes & & \\
\hline \hline
\end{tabular}

Notes: ${ }^{\dagger}$ Features not shown in the CloudyFIX column are the same as CloudyVAR. 


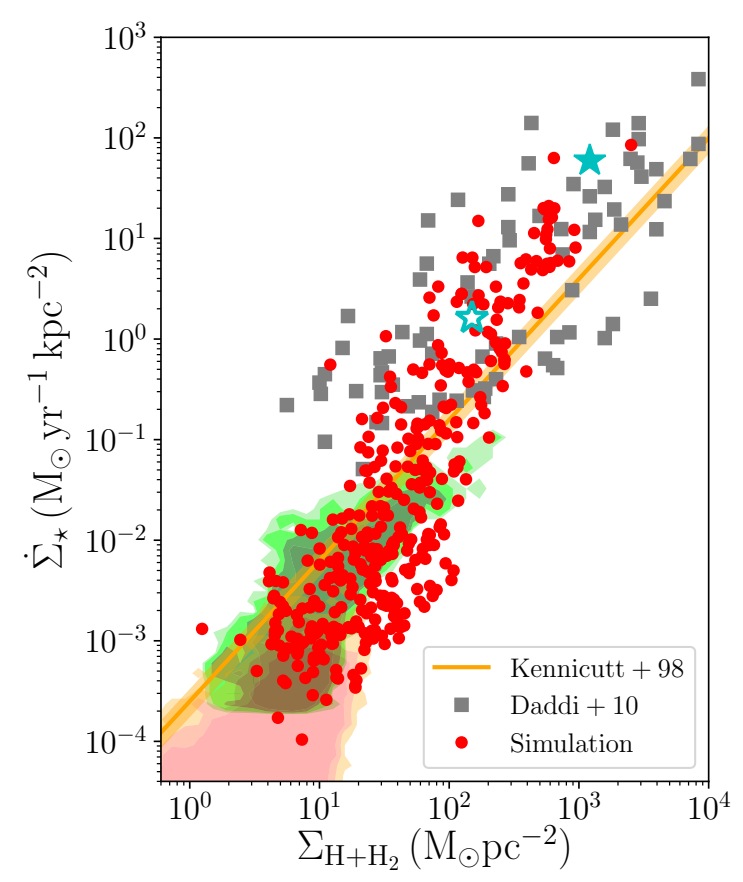

Figure 4. KS relation in the galaxy, shown as red points, compared with local data by Bigiel et al. (2010) (red/green contours), the best-fit by Kennicutt (1998) (orange shaded area and solid line), and the SB/SMG/ULIRG data reported in Daddi et al. (2010). The star symbols correspond to the average values for our galaxy, averaged over $2 \mathrm{kpc}$ (empty cyan star) or within the $\mathrm{H}_{2}$ half-mass radius for the gas and the stellar half-mass radius for stars (filled cyan star). The agreement with observations is very good across the entire range, with also the kink around $\Sigma_{\mathrm{H}+\mathrm{H}_{2}}=10 \mathrm{M}_{\odot} \mathrm{pc}^{2}$ well reproduced. At high densities $\left(\Sigma_{\mathrm{H}+\mathrm{H}_{2}} \gtrsim 30 \mathrm{M}_{\odot} \mathrm{pc}^{2}\right)$, we observe the transition towards the SB region for a few patches, in perfect agreement with the data by Daddi et al. (2010).

work we consider three different models, two based on CLOUDY (Ferland et al. 2017), i.e. model 'CloudyFIX' and model 'CloudyVAR', and one directly based on the chemical state of the gas in our simulation determined by KROME (model 'Krome'), as detailed in the following Sections.

We stress that the Krome model relies on the selfconsistent non-equilibrium chemical evolution within the cosmological simulation, where thermodynamics and chemistry are fully coupled, while this is not the case for CloudyFIX and CloudyVAR, that are simply applied to the outputs.

\subsubsection{Extracting FIR lines from KROME}

Thanks to our non-equilibrium chemical network, we can directly follow the abundances of different ions in our simulation, that are self-consistently evolved during the run in a fully-coupled fashion together with the thermodynamic state of the gas. The abundances of each species are tracked for all cells in the simulation, and correspond to the average abun- dances within the cell. ${ }^{5}$ In particular, heating and cooling processes of the gas are tightly bound to the instantaneous abundances of the species, and the temperature evolution directly affects the dynamics of the system. This allows us to accurately describe the ISM out-of-equilibrium conditions, in particular when the gas is affected by shocks ${ }^{6}$; since such conditions are likely to occur for gas below $T=10^{4} \mathrm{~K}$ (e.g. Bovino et al. 2016), this makes it hard to model them with a separate post-processing of the simulation results, especially since all these processes are tightly coupled.

Among the species included in our chemical network, we focus here on the line emission by two of the main coolants of the ISM, $\mathrm{C}^{+}$(whose cooling is dominated by the $157.7 \mu \mathrm{m}$ transition) and $\mathrm{O}$ (for which two transitions are considered, at $63 \mu \mathrm{m}$ and $146 \mu \mathrm{m}$ respectively), similarly to what described in Glover \& Jappsen (2007) and Grassi et al. (2014). To compute the intensity of the lines, KROME assumes a statistical equilibrium of the atomic excited and ground states (including collisional excitation and de-excitation, spontaneous and stimulated emission, and photon absorption). At the low frequencies typical of the FIR lines, we can safely assume that the only relevant radiation is the Cosmic Microwave Background (CMB), that can alter the state populations and reduce the emission at low density, in particular in high-redshift galaxies above $z \sim 4.5$, when the CMB temperature reaches a few tens of K (Da Cunha et al. 2013; Vallini et al. 2015). As an example, the equilibrium conditions can be written, for, e.g. a three-level ion like neutral $\mathrm{O}$, as

$\left[\begin{array}{ccc}1 & 1 & 1 \\ T_{01}+T_{02} & -T_{10} & -T_{20} \\ -T_{01} & T_{10}+T_{12} & -T_{21}\end{array}\right]\left[\begin{array}{c}n_{0} \\ n_{1} \\ n_{2}\end{array}\right]=\left[\begin{array}{c}n_{\mathrm{O}} \\ 0 \\ 0\end{array}\right]$

where $T_{i j}=C_{i j}+B_{i j} I_{v_{j i}}$ and $T_{j i}=C_{j i}+A_{j i}+B_{j i} I_{v_{j i}}$, with $j>$ $i=0,1,2$ the state indices, $C_{i j}\left(C_{j i}\right)$ the collisional excitation (de-excitation) rate, $A_{j i}$ the spontaneous emission rate, $B_{i j}$ $\left(B_{j i}\right)$ the absorption (stimulated emission) rate, and $I_{v_{j i}}$ the radiation intensity at the transition frequency $v_{j i}$. From the state populations obtained, we then compute the emissivity of each line as

$\Lambda_{\text {net }}=\Lambda-\Gamma=n_{j}\left(A_{j i}+B_{j i} I_{v_{j i}}\right)-n_{i} B_{i j} I_{v_{j i}}$

\subsubsection{Post-processing the simulation with CLOUDY}

An alternative to our non-equilibrium approach, that is typically employed for simulations where chemical species abundances are not directly evolved, is the post-processing with CLOUDY, that we describe in the following.

In CLOUDY models, each cell of the simulation is assumed to be a homogeneous slab, which is then split in a multiple optically-thin layers for which species abundances, temperature, and line emission can be computed assuming photo-ionisation equilibrium conditions, and accounting for

5 This implies that chemistry is solved assuming an optically thin slab, and photon absorption is then applied on the entire cell size to update the radiation flux transmitted to adjacent cells.

${ }^{6}$ With the term shocks we consider each hydrodynamic interaction in which $v_{\text {gas }} \gg c_{s}$, and not only those that produce extremely hot gas with $T \gg 10^{5} \mathrm{~K}$. 
radiation absorption throughout the slab itself. Different choices can be made for the temperature throughout the slab, which can be kept fixed at a desired value or let free to evolve according to heating and cooling processes. Here we consider both cases, that we name CloudyFIX and CloudyVAR, respectively.

For both models, we generate a grid of CLOUDY models and interpolate the simulation data over the resulting multidimensional table, as a function of different parameters. For the impinging flux, we assume the SED of a entire stellar population based on the updated Bruzual \& Charlot (2003) models. Similarly to Pallottini et al. (2019), we consider the full SED with its ionising component $\left(E_{\gamma}>13.6 \mathrm{eV}\right)$ only for particles having an ionising parameter $U>10^{-4}$, while a non-ionising radiation field is considered for the other particles.

In model CloudyVAR, our grid is a function of the total gas density $\rho$, the gas metal fraction $f_{\mathrm{Z}}$, the UV radiation field $G$ (in units of the Milky-Way value $G_{0}$ ), and the total gas column density $N_{\text {gas }}$. The gas temperature is allowed to vary throughout the slab according to the heating and cooling rates computed by CLOUDY, including photo-heating. This means that we lose consistency with the thermodynamic state determined in the simulation. Nevertheless, as we are limited by the resolution, and we cannot solve on-thefly the photodissociation region (PDR) structure, this is the only choice able to exploit the chemistry and temperature changes within the PDR.

In model CloudyFIX, we force CLOUDY to maintain a constant temperature throughout the slab: effectively $T$ is considered as an additional parameter in the grid, that is interpolated using the values obtained from the simulation. We notice that, in this case, the temperature of the slab is determined by full non-equilibrium evolution in the simulation, and does not necessarily corresponds to the temperature one would get by employing CLOUDY tables for cooling and heating (see Capelo et al. 2018, for details).

This method has already been used in other studies (e.g. Katz et al. 2019), as a way to mimic the presence of shocks (Egami et al. 2018), i.e. the photo-ionisation equilibrium assumption is only used to set the abundances (and the emission) at the chosen temperature (see Olsen et al. 2018; Pallottini et al. 2019, for further discussion).

Note that, in our estimate of the emission line luminosity with CLOUDY, we do not assume any unresolved structure for the cells, unlike it is done in Vallini et al. (2018) and Pallottini et al. (2019), to ensure a consistent comparison with the model 'Krome' emission.

Although these approaches are all valid, each of them has its own advantages and weaknesses that should properly be acknowledged. In Table 1, we summarise all the differences among the models. Keep in mind that these differences are only related to how the instantaneous line emission is derived for every cell in the simulation at the desired redshift, and do not consider any time evolution, which is instead computed with KROME according to the timedependent non-equilibrium chemistry and cooling/heating processes.

In particular, although CLOUDY models exhibit a much higher accuracy in the treatment of the chemistry, ionisation equilibrium is assumed and does (CloudyFIX) or does not (CloudyVAR) take into account any dynamical effect onto
Table 2. Total luminosity for the emission maps reported in Fig. 5 .

\begin{tabular}{|c|c|c|c|c|}
\hline Model & $\begin{array}{c}L_{[\mathrm{CII}]_{158 \mu}} \\
\left(\mathrm{L}_{\odot}\right)\end{array}$ & $\begin{array}{c}L_{[\mathrm{OI}]_{63 \mu} \mu} \\
\left(\mathrm{L}_{\odot}\right)\end{array}$ & $\begin{array}{c}L_{[\mathrm{OI}]_{146 \mu}} \\
\left(L_{\odot}\right)\end{array}$ & $\begin{array}{c}\left.L_{[\mathrm{OIII}}\right]_{88 \mu} \\
\left(\mathrm{L}_{\odot}\right)\end{array}$ \\
\hline CloudyFIX & $10^{7.47}$ & $10^{7.77}$ & $10^{6.84}$ & $10^{7.66}$ \\
\hline CloudyVAR & $10^{7.21}$ & $10^{6.24}$ & $10^{4.98}$ & $10^{7.65}$ \\
\hline Krome & $10^{7.71}$ & $10^{8.01}$ & $10^{6.86}$ & - \\
\hline
\end{tabular}

the gas temperature. On the other hand, KROME uses a simplified network and is tied to the simulation resolution, i.e. it cannot model PDRs unless they are properly resolved in the simulation. However, it naturally accounts for any deviations from ionisation equilibrium and dynamical effects naturally arising in the simulation.

\subsection{Predicted line emission}

In Fig. 5, we compare the emission maps of $[\mathrm{CII}]_{158 \mu},[\mathrm{OI}]_{63 \mu}$, and $[\mathrm{OI}]_{146 \mu}$ obtained with the three approaches reported in Table 1 . We consider also $[\mathrm{OIII}]_{88 \mu}$, for which we only report the results obtained with CLOUDY, since $\mathrm{O}^{++}$was not included in our KROME chemical network. The integrated luminosity over the maps are reported in Table 2.

The $[\mathrm{CII}]_{158 \mu}$ maps show that $\mathrm{C}^{+}$emission from model 'CloudyFIX' is stronger than that from 'CloudyVAR', especially in the spiral arms and the galaxy nucleus, but no significant differences are observed in the outskirts of the galaxy. In model Krome, instead, the $[\mathrm{CII}]_{158 \mu}$ emission in the outskirts is slightly suppressed relative to the CLOUDY models, but is enhanced in the spiral arms of the galaxy. Overall, the total luminosity differs by a factor of $\sim 3$ between the smallest (CloudyVAR) and the largest value (Krome).

For $[\mathrm{OI}]_{63 \mu}$ and $[\mathrm{OI}]_{146 \mu}$ the differences are much larger (a factor of $\sim 59$ and $\sim 76$, respectively). Models CloudyFIX and Krome predict a similarly strong emission from the dense gas in the spiral arms and a very weak emission from the low-density gas, always within a factor of two from each other (interestingly, $[\mathrm{OI}]_{146 \mu}$ is almost identical in the two models), whereas model CloudyVAR exhibits up to two orders of magnitude weaker emission across the entire galaxy. In the galaxy nucleus, this difference becomes larger, with [OI] emission being more centrally concentrated in models CloudyFIX and Krome, and almost negligible in CloudyVAR. Because of the stronger dependence of [OI] emission on the gas temperature, the differences we find can be easily explained with the typically lower temperatures predicted in the CloudyVAR model relative to those in the simulation.

For $[\mathrm{OIII}]_{88 \mu}$ emission, the differences between CloudyFIX and CloudyVAR are mild, with the emitting region being almost identical in the two cases. A possible explanation for this result is that, because of the high ionisation energy of $\mathrm{O}^{+}(35 \mathrm{eV})$, both $\mathrm{SNe}$ and ionising radiation can efficiently produce $\mathrm{O}^{++}$; however, the gas shock-heated by SNe has typically low densities, while $[\mathrm{OIII}]_{88 \mu}$ traces denser gas. Hence, the only plausible responsible for $[\mathrm{OIII}]_{88 \mu}$ emission remains the ionising radiation from young massive stars, which is typically absorbed in the outer layers of the slab, almost independently of the actual gas temperature. 


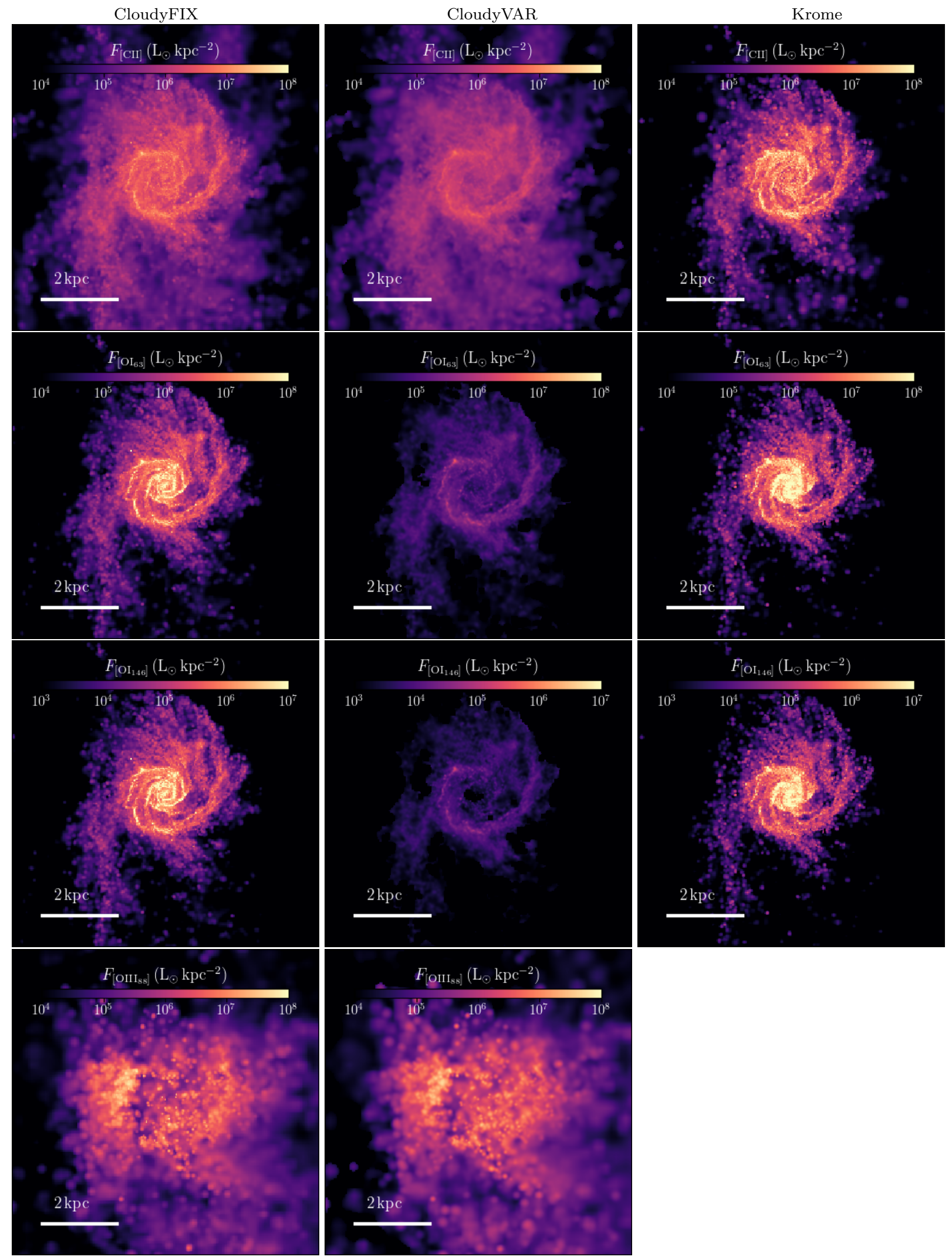

Figure 5. Emission maps of the galaxy at $z=6$ obtained from our different models, i.e. CloudyFIX (left panels), CloudyVAR (middle panels), and Krome (right panels). While for $[\mathrm{CII}]_{158 \mu}$ and $[\mathrm{OIII}]_{88 \mu}$ lines the assumption made for the cell temperature in CLOUDY is not important, resulting in moderate differences, for [OI] lines the emission varies by up to two orders of magnitude. For model Krome, we find still different results, but closer to model CloudyFIX than to CloudyVAR, because of the identical choice in the temperature of the cell. 

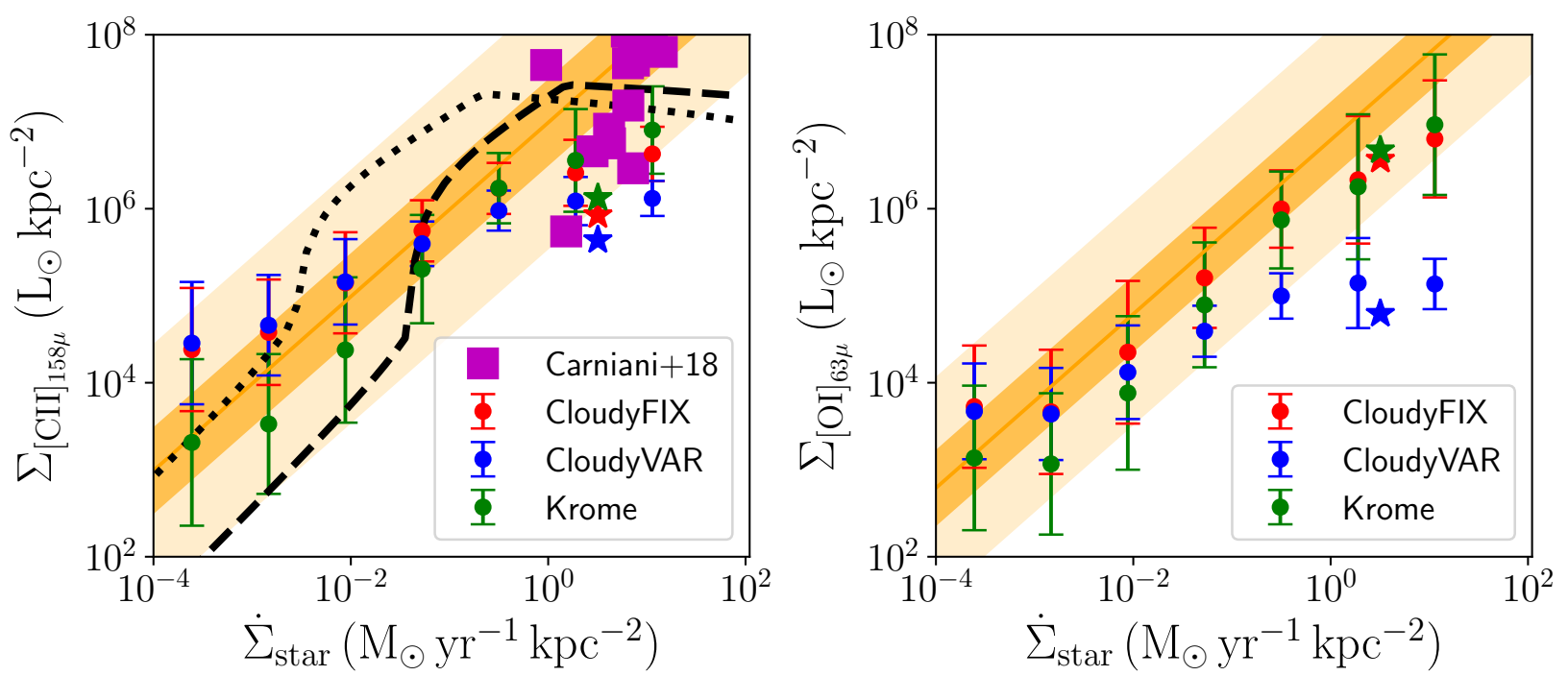

Figure 6. $[\mathrm{CII}]_{158 \mu}-\mathrm{SFR}$ (left-hand panel) and $[\mathrm{OI}]_{63 \mu}-\mathrm{SFR}$ (right-hand panel) correlations for the three different models applied to our simulation at $z=6$, compared with the results by De Looze et al. (2014), shown as an orange solid line and the two shaded areas, the smallest corresponding to $1-\sigma$ and the largest to $3-\sigma$, and the theoretical model by Ferrara et al. (2019) for $Z=0.5 \mathrm{Z}_{\odot}$ and $k_{\mathrm{s}}=1$ (black dotted line) and $k_{\mathrm{s}}=5$ (black dashed line). The coloured stars correspond to the average value for our models if the galaxy is observed at $2.5 \mathrm{kpc}$ resolution. All models reproduce the $[\mathrm{CII}]_{158 \mu}-\mathrm{SFR}$ correlation reasonably well, with moderate differences in the slope. On the other hand, the $[\mathrm{OI}]_{63 \mu}-\mathrm{SFR}$ correlation can be reproduced only by Krome and CloudyFIX, with CloudyVAR predicting a strong deficit at high SFR surface densities.

As a further comparison, we also investigate where the galaxy lies relative to the correlations between $[\mathrm{CII}]_{158 \mu}$ (and $[\mathrm{OI}]_{63 \mu}$ ) and the SFR observed at low (and high) redshift (De Looze et al. 2014). For this analysis, we extract the FIR fluxes from the maps in Fig. 5, degraded to 300 pc resolution, and the SFR surface density from an equivalent map of the intrinsic FUV emission of the galaxy, applying the conversion factor in Salim et al. (2007) for a Kroupa IMF. The results are shown in Fig. 6, where the left panel corresponds to the $[\mathrm{CII}]_{158 \mu}-\mathrm{SFR}$ correlation and the right panel to the $[\mathrm{OI}]_{63 \mu}-\mathrm{SFR}$ counterpart. The simulation data have been binned in 8 bins about 1-dex wide, and bins with less than 5 data points have been excluded to avoid any bias in the measure. The average value for the entire galaxy, is observed at $2.5 \mathrm{kpc}$ resolution, is shown as coloured stars, using the colour corresponding to each model. The orange solid line and the two shaded areas correspond to the best-fit relation by De Looze et al. (2014) on the entire literature sample, assuming a perfectly linear slope, and its $1-\sigma$ (smallest) and $3-\sigma$ (largest) uncertainty. We also show the theoretical results by Ferrara et al. (2019) as black lines, obtained assuming a gas metallicity $Z=0.5 \mathrm{Z}_{\odot}$ and a SFR lying $k_{\mathrm{S}}$ times off the Schmidt-Kennicutt relation, with $k_{S}=1$ (dotted line) and $k_{\mathrm{S}}=5$ (dashed line).

For the $[\mathrm{CII}]_{158 \mu}$ relation, our results (independent of the model considered) are slightly offset relative to the local relation, but consistent with observational results of highredshift galaxies (see, e.g. Carniani et al. 2018). Nevertheless, there are small differences in the slope of the relation. At low SFRs $\left(\dot{\Sigma}_{\text {star }} \lesssim 1 \mathrm{M}_{\odot} \mathrm{yr}^{-1}\right)$, Krome well matches the observed slope (as already shown in Lupi \& Bovino 2020), whereas the slope in models CloudyFIX and CloudyVAR is moderately shallower, with the data lying above the best- fit relation. At high SFRs $\left(\dot{\Sigma}_{\text {star }} \gtrsim 1 \mathrm{M}_{\odot} \mathrm{yr}^{-1}\right)$, all models predict a saturation of the emission, consistent with the predictions by Ferrara et al. (2019). Among the models, CloudyVAR exhibits the lowest saturation value, whereas CloudyFIX and Krome lie slightly above. This is due to the typically lower temperature in CloudyVAR for the higherdensity cells, that likely correspond to the more star-forming regions of the galaxy. Relative to the model by Ferrara et al. (2019), Krome better follows the $k_{\mathrm{s}}=5$ case, although it does not show the enhanced $[\mathrm{CII}]_{158 \mu}$ luminosity around $\dot{\Sigma}_{\text {star }}=0.1 \mathrm{M}_{\odot} \mathrm{yr}^{-1} \mathrm{kpc}^{-2}$, and then saturates around $\dot{\Sigma}_{\text {star }}=1 \mathrm{M}_{\odot} \mathrm{yr}^{-1} \mathrm{kpc}^{-2}$. On the other hand, both CLOUDY models exhibit a higher luminosity at low SFRs, more consistent with the $k_{\mathrm{S}}=1$ case, and then deviate towards the $k_{\mathrm{s}}=5$ case, finally saturating at a SFR similar to that observed for Krome. The average values, that are dominated by the central patches where the relation saturates, exhibit a deficit relative to the local relation, but are still reasonably consistent with Carniani et al. (2018).

For [OI] lines, the differences are more noticeable. Models CloudyFIX and Krome are quite similar, and follow reasonably well the observed slope (also when the average values are considered). Model CloudyVAR produces instead a much lower emission from dense gas, resulting in a very shallow relation and significant deviations at large SFR surface densities, also reflected in the average value.

Observationally, lines ratios such as $[\mathrm{OI}]_{63 \mu} /[\mathrm{CII}]_{158 \mu}$ and $[\mathrm{OIII}]_{88 \mu} /[\mathrm{CII}]_{158 \mu}$ have been widely use to investigate the ISM properties. In particular, $[\mathrm{CII}]_{158 \mu}$ is emitted in both cold neutral medium and PDRs, $[\mathrm{OI}]_{63 \mu}$ by dense gas and warm PDRs (as long as other mechanisms like mechanical or X-ray heating do not dominate), and $[\mathrm{OIII}]_{88 \mu}$ by the ionised gas near stellar 

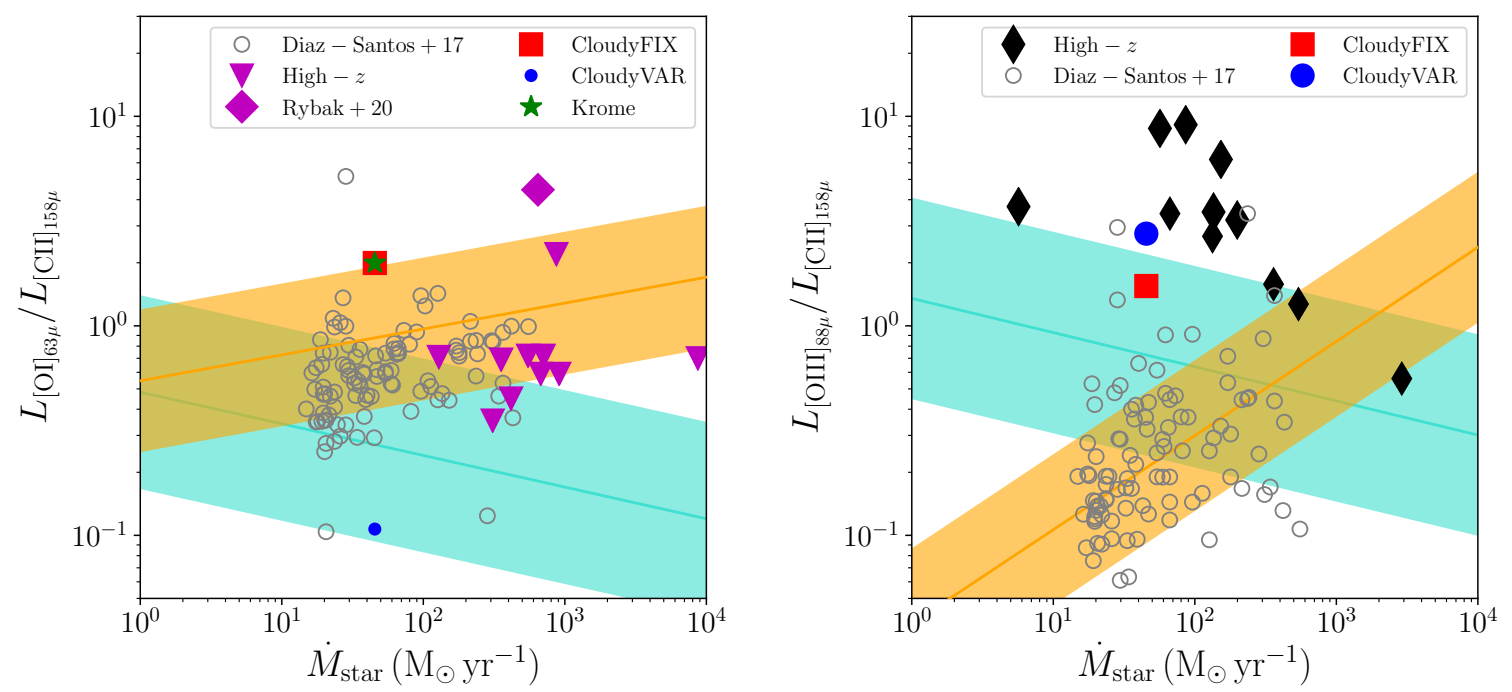

Figure 7. FIR line ratios for our different models, compared with local observations by De Looze et al. (2014) (orange shaded area), Díaz-Santos et al. (2017) (grey circles), and high-redshift galaxies by Carniani et al. (2017); Walter et al. (2018); Marrone et al. (2018); Hashimoto et al. (2019); Harikane et al. (2019) (black diamonds), Brisbin et al. (2015); Zhang et al. (2018) (magenta triangles), and Rybak et al. (2020) (the magenta diamond in the left-hand panel). The red square corresponds to model CloudyFIX, the blue dot to CloudyVAR, and the green star to Krome. For the $[\mathrm{OI}]_{63 \mu}-[\mathrm{CII}]_{158 \mu}$ ratio, all models but CloudyVAR agree well with each other and observations. CloudyVAR, by predicting a very weak $[\mathrm{OI}]_{63 \mu}$ emission, results in a mild discrepancy with local data, and an even stronger one with other high-redshift data. In the right-hand panel, instead, CloudyVAR agrees better with observations, whereas CloudyFIX results in a factor of two lower ratio, smaller than typically observed values at the same SFR.

sources. Hence, the $[\mathrm{OI}]_{63 \mu}-[\mathrm{CII}]_{158 \mu}$ ratio represents a good tracer of the typical ISM density and PDR temperature, whereas the $\left.[\mathrm{OIII}]_{88} \mu^{-[\mathrm{CII}}\right]_{158 \mu}$ gives us information about the ionisation parameter in the ISM and/or the PDR filling factor. Fig. 7 shows these ratios for our galaxy, compared to high-redshift observations. In the left-hand panel, we see that all models but CloudyVAR appear moderately consistent with local starbursts by De Looze et al. (2014) and Díaz-Santos et al. (2017), with the ratio being in the upper end of the observed range. Relative to high-redshift galaxies, our simulation lies in between the $z \sim 1-4$ and the $z \sim 6$ data. CloudyVAR, on the other hand, because of the extremely weak $[\mathrm{OI}]_{63 \mu}$ emission, results in an extremely low $[\mathrm{OI}]_{63 \mu} /[\mathrm{CII}]_{158 \mu}$ ratio, lying at the lower end of the local dwarf region. The $[\mathrm{OIII}]_{88 \mu} /[\mathrm{CII}]_{158 \mu}$ ratio varies by about a factor of two between the two CLOUDY models, with CloudyVAR in this case being closer to the observed high-redshift data with respect to CloudyFIX. Compared to the local relations by De Looze et al. (2014), our simulation (for both models) agrees better with the dwarf galaxy correlation rather than with starbursts by De Looze et al. (2014), although it is still compatible with the highest data points of the LIRG sample by Díaz-Santos et al. (2017).

\subsection{Phase diagrams and ion abundances}

In order to better constrain the origin of the differences in luminosity, we inspect the typical thermodynamic conditions of the gas responsible for the emission. In Fig. 8, we show the density-temperature diagrams for our galaxy at $z=6$, weighted by the $[\mathrm{CII}]_{158 \mu}$ (top row), $[\mathrm{OI}]_{63 \mu}$ (middle row), and $[\mathrm{OI}]_{146 \mu}$ (bottom row) luminosity.

For $[\mathrm{CII}]_{158 \mu}$, all models are reasonably similar, apart from the warm high-density region where Krome predicts a factor of a few higher luminosity compared to CloudyFIX and CloudyVAR. Because of the variable temperature across the slab, CloudyVAR predicts the lowest luminosity among the models, but still reasonably consistent with them (within a factor of $\sim 3$ ). Interestingly, CLOUDY models give a moderately higher luminosity at low densities relative to Krome, likely because of the assumption of ionisation equilibrium, which is harder to reach (see Oppenheimer \& Schaye 2013; Richings et al. 2014; Bovino et al. 2016, for a discussion).

For [OI] lines, instead, the differences are much larger, with Krome and CloudyFIX exhibiting an emission peak in warm, high-density gas compared to CloudyVAR. As for $[\mathrm{CII}]_{158 \mu}$, Krome shows the highest luminosity in this regime (a factor of a 2-3 higher compared to CloudyFIX). However, the $[\mathrm{OI}]$ emission in CloudyFIX at very high densities $\left(n_{\mathrm{H}_{\mathrm{tot}}}>100 \mathrm{~cm}^{-3}\right)$ is somewhat larger than that in Krome, suggesting that $[\mathrm{OI}]$ emission in this regime is stronger in photoionisation equilibrium conditions. CloudyVAR, instead, exhibits extremely low luminosities for [OI] lines, likely because the temperature within the slab drops quickly to low values where $\mathrm{O}$ is not efficiently excited.

In order to understand the reason for the higher luminosity in Krome for gas in the range $10 \mathrm{~cm}^{-3}<\mathrm{n}_{\mathrm{H}_{\mathrm{tot}}}<$ $1000 \mathrm{~cm}^{-3}$ compared to CloudyFIX, we checked the abundance of $\mathrm{CO}$ in the corresponding density range with CLOUDY, finding that a non-negligible part of $\mathrm{C}$ and $\mathrm{O}$ are indeed bound into CO (see, e.g, Glover \& Jappsen 2007, for a 


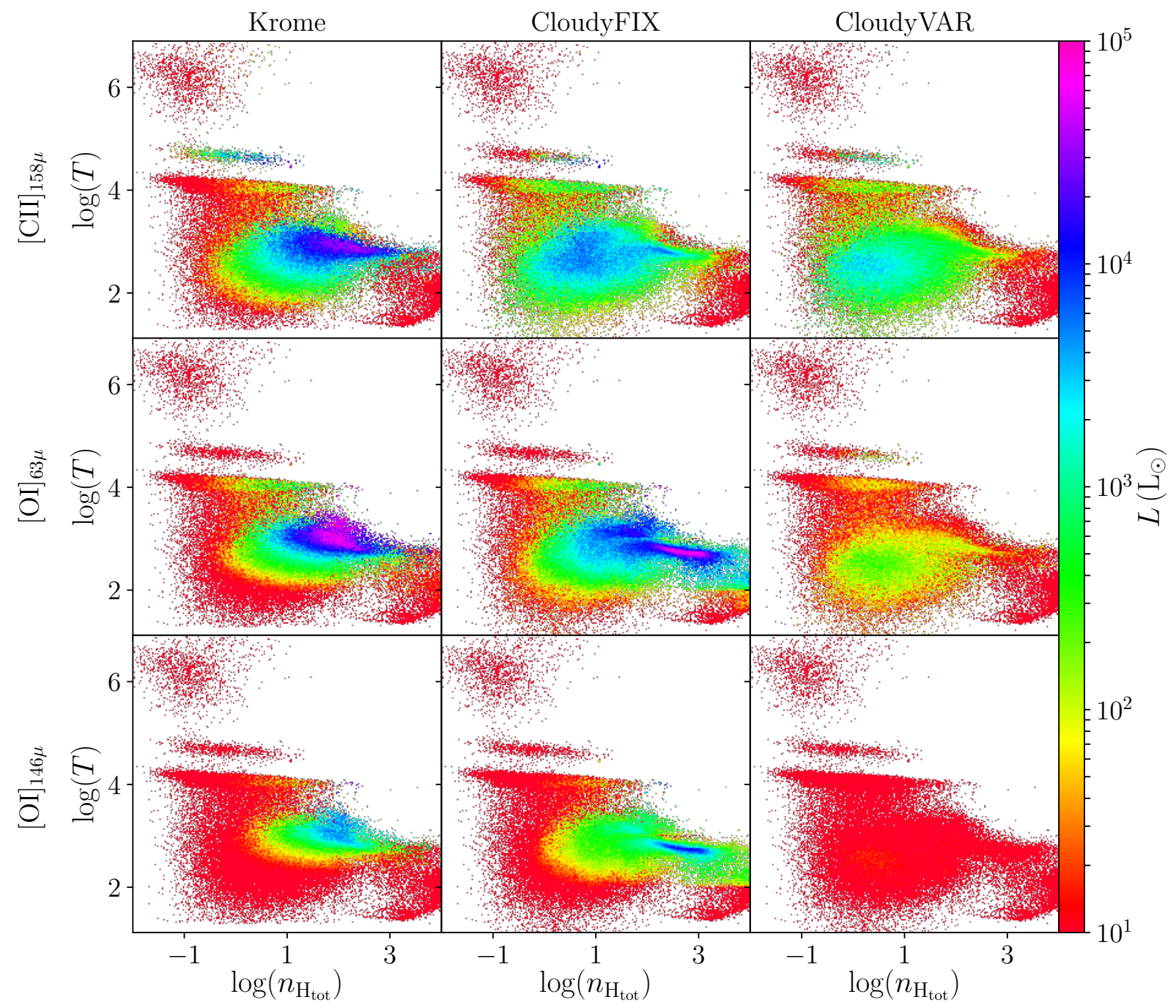

Figure 8. Density-temperature diagram for our three models, weighted by the FIR line luminosity. The first row corresponds to $[\mathrm{CII}]_{158 \mu}$, the middle one to $[\mathrm{OI}]_{63 \mu}$, and the bottom one to $[\mathrm{OI}]_{146 \mu}$, with Krome shown in the left-most column, CloudyFIX in the middle one, and CloudyVAR in the right-most one. $[\mathrm{CII}]_{158 \mu}$ luminosity is reasonably similar in all models, with the only noticeable differences being a higher peak in Krome at high density, and a globally lower emission in CloudyVAR. For [OI] lines, CloudyVAR results in extremely low emission, consistent with the very low line ratio in Fig. 7, whereas Krome and CloudyFIX give more similar results, with the stronger difference resulting from the high-density region. At low densities, CLOUDY models give somewhat larger luminosities for all lines compared to Krome. A similar discrepancy is found at very high densities, where no emission is obtained with Krome, whereas CloudyFIX produces a non-negligible fraction of the line luminosity.

discussion), not included in our chemical network. Although, in principle, such a difference could also arise because of the $[\mathrm{OI}]_{63 \mu}$ line becoming thick in the inner layers of the cell/slab (where we reach $A_{\mathrm{V}} \sim 3-4$; see, Kaufman et al. 1999 for a discussion), an effect not modelled in Krome, this discrepancy should increase at even higher densities, where $A_{\mathrm{V}}$ is larger (see, e.g., Grassi et al. 2017). However, this is not seen in our case, where at $n_{\mathrm{H}_{\mathrm{tot}}} \gtrsim 10^{3} \mathrm{~cm}^{-3}$ CloudyFIX predicts larger luminosities compared to Krome, we can safely assume this effect does not play a significantly role on our results. At low-densities, instead, the higher $[\mathrm{CII}]_{158 \mu}$ and $[\mathrm{OI}]_{63 \mu}$ luminosities in Krome are due to the higher ionisation states, not included in our network (see, for instance, the hot gas where $\mathrm{C}^{++}$should form).

To assess whether these differences arise from the different ion abundances or are linked to the determination of the gas temperature and the photoionisation conditions, we show in Fig. 9 phase diagrams weighted by the $\mathrm{C}, \mathrm{C}^{+}, \mathrm{O}$, and 


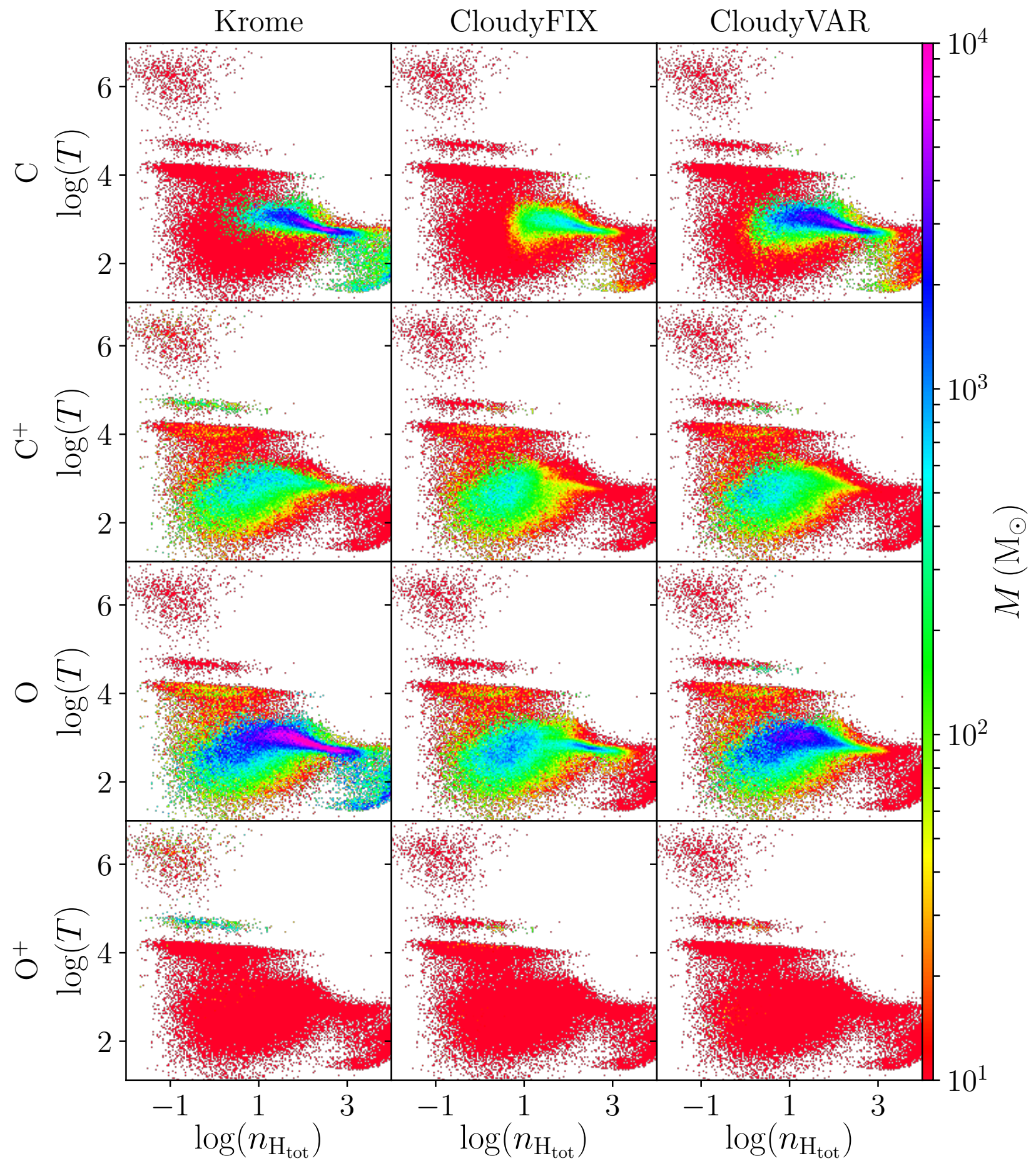

Figure 9. Same as Fig. 8, weighted by the ion abundance. All models look similar in terms of $\mathrm{O}^{+}$and $\mathrm{C}^{+}$masses, with mild differences due to the missing $\mathrm{CO}$ in Krome and to possible small deviations from photoionisation equilibrium. Neutral species are instead more affected by the different thermodynamic assumptions in the models. Krome, because of the missing $\mathrm{CO}$, produces too much $\mathrm{O}$ and $\mathrm{C}$ at high densities $\left(n_{\mathrm{H}, \text { tot }}>10 \mathrm{~cm}^{-3}\right)$, although similar abundances for $\mathrm{C}$ are found in CloudyVAR as well. CloudyFIX, instead, results in lower abundances of these ions, because of the more efficient conversion to $\mathrm{CO}$ at $T \sim 500-1000 \mathrm{~K}$. 
$\mathrm{O}^{+}$abundances. The first column shows the mass of each species in our simulation, as obtained by KROME, whereas the second and third ones correspond to models CloudyFIX and CloudyVAR, respectively.

$\mathrm{C}$ and $\mathrm{C}^{+}$behave in a reasonably similar way in all models, with neutral $\mathrm{C}$ dominating the high-density tail of the gas, even at $T \sim 1000 \mathrm{~K}$, and $\mathrm{C}^{+}$being the most important ion for lower density gas, at all temperatures up to a few $10^{4} \mathrm{~K}$. While the agreement among the models for $\mathrm{C}^{+}$is very good, with only mild differences consistent with those observed in the luminosity diagrams (within a factor of two), C is more significantly affected by the model choice, with total masses that differ by up to a factor of 7 (between the two CLOUDY models). In this case, we observe an opposite behaviour compared to Fig. 8, with Krome and CloudyVAR showing similar abundances, whereas CloudyFIX predicts a lower abundance of neutral C, probably due to a more efficient formation of $\mathrm{CO}$ in this case.

$\mathrm{O}^{+}$is abundant only in warm/hot gas above $10^{4} \mathrm{~K}$ in Krome, and is almost completely missing in CloudyFIX and CloudyVAR, due to its conversion to $\mathrm{O}^{++}$or even higher ionisation states ${ }^{7}$. Neutral $\mathrm{O}$ is instead predominant everywhere in the galaxy ISM in all models. For O, the agreement is reasonably good among all models, with the largest differences appearing in the CO-forming region $\left(10 \lesssim n_{\mathrm{H}_{\mathrm{tot}}} /\left(\mathrm{cm}^{-3}\right) \lesssim 10^{3}\right.$ and $\left.T \sim 3000 \mathrm{~K}\right)$. Surprisingly, also in this case Krome agrees better with CloudyVAR than with CloudyFIX.

We conclude that, rather than the actual metal ion abundances, temperature plays a major role in determining the emission. This is because $T$ regulates both the species abundances and the excitation cross sections, hence affecting the line emission efficiency. As a consequence, even lower abundances of the emitting ion can result in a larger luminosity.

\section{CAVEATS}

Before moving to the conclusions, there are a few important caveats that should be considered.

\subsection{Uncertainties of the KROME model}

First, our non-equilibrium approach, by not including the formation and dissociation of $\mathrm{CO}$, might overestimate the emission of neutral atoms like $\mathrm{O}$, especially at high density. Although the inclusion of CO could improve significantly the prediction capabilities of our approach, the additional cost of a CO network would have made our cosmological simulations much more computationally expensive to perform (we would need at least $\sim 300$ reactions as in Grassi et al. 2017 against the $\sim 85$ employed here), and its inclusion is deferred to future studies. Another possible issue is related to the $[\mathrm{OI}]_{63 \mu}$ emission, because of the pathological nature of this line, which can often become optically thick (Kaufman

\footnotetext{
7 Since both in KROME and CLOUDY we assume solar ratios for the metal abundances, the total amount of oxygen nuclei is expected to be the same in all the three models, and the only differences are due to the relative abundance of different ionisation states and/or the formation of oxygen-bearing molecules.
}

et al. 1999), a regime not included in our cell-average approach with KROME. Nevertheless, the optical depth in our $[\mathrm{OI}]_{63 \mu}$-luminous cells is not extremely large, and this effect is most likely marginal relative to $\mathrm{CO}$ conversion.

Secondly, the chemical network adopted in this study does not include highly ionised species like $\mathrm{C}^{++}$and $\mathrm{O}^{++}$. These species could in principle decrease the amount of $\mathrm{C}^{+}$and $\mathrm{O}^{+}$in the ISM, and slightly suppress the emission, giving results less accurate than with CLOUDY. However, our analysis showed that the typical conditions for double (at least) ionisation, i.e. very hot gas or the presence of hard radiation, are not typical of the ISM of star-forming galaxies, hence our approximation does not significantly affect our conclusions.

Third, chemistry is limited by the simulation resolution when KROME is employed, and any possible sub-grid distribution cannot be accounted for.

\subsection{Uncertainties of the CLOUDY models}

Caveats are also related to the post-processing performed with CLOUDY. The first and most important is that the radiative flux extracted from the cell and passed to CLOUDY is already processed in the simulation, hence could be already attenuated compared to the intrinsic flux impinging on the cell and actually affecting the chemistry.

Secondly, if we let CLOUDY recompute the temperature within the slab, we risk neglecting gas shocks and getting a temperature that is not fully consistent with the one in the simulation. Although the temperature distribution through the slab could be more realistic in equilibrium conditions, this is not consistent with the hydrodynamics we rely upon. If, instead, we keep the temperature constant through the slab at the value obtained in the simulation, we are more consistent with the simulation, but we do not properly consider how the gas temperature would evolve through the slab because of radiation absorption. Thirdly, we have to keep in mind that CLOUDY is reliable as long as the gas is in photo-ionisation equilibrium and no significant deviations from occur in the gas (Richings et al. 2014; Richings \& Schaye 2016), and this strongly depends on the dynamical evolution of the system under scrutiny, so particular care should be taken. At last, we have to consider that in most simulations, where chemistry is not coupled with the hydrodynamics, cooling and heating are computed according to CLOUDY calculations including (or not) a uniform UV background, but neglecting the interstellar radiation field, which is instead included in subsequent emission line calculations, producing possibly inconsistent results.

\section{DISCUSSION AND CONCLUSIONS}

In this work, we presented a state-of-the-art cosmological simulation of a star forming galaxy at high-redshift that includes, for the first time, on-the-fly radiative transfer (in ten bins ranging from $0.75 \mathrm{eV}$ up to $1 \mathrm{keV}$ ) and non-equilibrium chemistry for the primordial $\left(\mathrm{H}, \mathrm{H}^{+}, \mathrm{H}^{-}, \mathrm{He}, \mathrm{He}^{+}, \mathrm{He}^{++}\right.$, $\mathrm{H}_{2}$, and $\left.\mathrm{H}_{2}^{+}\right)$and the main metal species $\left(\mathrm{C}, \mathrm{C}^{+}, \mathrm{O}, \mathrm{O}^{+}, \mathrm{Si}\right.$, $\mathrm{Si}^{+}$, and $\left.\mathrm{Si}++\right)$. Our subgrid model is an improved version of the one presented in Lupi \& Bovino (2020). Our simulation predicts an evolved galaxy with already roughly so- 
lar metallicity and well developed stellar and gaseous discs, similar to previous results (Pallottini et al. 2019; Katz et al. 2019). Thanks to the extremely high spatial ( 3 pc) and mass $\left(2 \times 10^{4} \mathrm{M}_{\odot}\right.$ per baryonic particle) resolution, and the coupling with KROME (Grassi et al. 2014), we were able to investigate the impact of non-equilibrium thermochemistry on the system evolution and to assess its effect on the Far Infrared (FIR) emission lines. We compared our nonequilibrium treatment with CLOUDY models applied in postprocessing on the simulation (Vallini et al. 2017; Pallottini et al. 2019), making different assumptions about the thermal structure within each cell. This analysis allowed us to constrain the uncertainty in the predicted FIR line luminosity and flux spatial distribution coming from different theoretical models.

Our results show that, despite the huge conceptual differences in the three approaches we considered, the $[\mathrm{CII}]_{158 \mu}$ luminosity is not strongly affected by our choice (up to a factor of 3); hence, the estimates can be considered reliable and be used as a tool to infer the ISM properties in observed galaxies. As a consequence, we can safely consider $[\mathrm{CII}]_{158 \mu}$ as a good tool to constrain the physics included in the simulations, that could help us better constraining our understanding of galaxy formation.

On the other hand, [OI] lines are much more affected by line model choice, which translates into a less predictive power of the hydrodynamic simulations. For instance, the $[\mathrm{OI}]_{63 \mu} /[\mathrm{CII}]_{158 \mu}$ ratio inf Fig. 7 either predicted, according to the chosen model, a dense/warm ISM or a low-density and cold one. Interestingly, this makes [OI] lines crucial to disentangle the models and assess which assumptions about the thermodynamic state of the gas better reproduce the ISM of high-redshift galaxies.

In addition to $[\mathrm{CII}]_{158 \mu}$ and $[\mathrm{OI}]$ lines, $[\mathrm{OIII}]_{88 \mu}$ represents an important tracer of the ionisation state of the gas around stellar sources, and is becoming more and more important in observations. Although not included in our current chemical network, we compared the two CLOUDY approaches, finding that $[\mathrm{OIII}]_{88 \mu}$ emission is not significantly affected by the temperature assumption, but only by the ionising flux reaching the cloud, and the luminosity is consistent with observations. This makes the $[\mathrm{OIII}]_{88 \mu}$ line a powerful tool to investigate the ionisation state of the ISM, almost independently of its thermo-dynamic state. However, particular care needs to be taken with the $[\mathrm{OIII}]_{88 \mu} /[\mathrm{CII}]_{158 \mu}$ ratio, since high values could either support the case of a higher ionisation parameter/lower covering fraction of PDRs, or simply a less efficient $[\mathrm{CII}]_{158 \mu}$ emission.

Concluding, the good agreement between Krome and CloudyFIX we found seems to suggest that gas in our simulated galaxy is not always far from equilibrium, hence that tabulated CLOUDY calculations represent an accurate and 'cheaper' alternative to on-the-fly chemistry. Nevertheless, we must notice that the input temperature in CLOUDY has been computed via a self-consistent non-equilibrium modelling of the chemistry of both primordial and metal species and all the relevant heating/cooling processes of the gas, and not via equilibrium tables where the effect of a variable radiation flux is not accounted for. This means that the temperature distribution we consider is more accurate than that we would have obtained employing equilibrium tables, and, as a consequence, the emission line calculations as well. Therefore, we stress that a proper inclusion of nonequilibrium chemistry calculations in simulations as done by KROME is crucial to get a better understanding of the interplay between microphysics and dynamics, and of galaxy formation and evolution in general, as also shown by Richings \& Schaye (2016) and Capelo et al. (2018).

\section{ACKNOWLEDGEMENTS}

$\mathrm{AL}, \mathrm{AF}$, and SC acknowledge support from the European Research Council No. 740120 'INTERSTELLAR'. This work reflects only the authors' view and the European Research Commission is not responsible for information it contains. Support from the Carl Friedrich von SiemensForschungspreis der Alexander von Humboldt-Stiftung Research Award is kindly acknowledged (AF). SB thanks for funding through PCI Redes Internacionales project number REDI170093, Conicyt PIA ACT172033 and BASAL Centro de Astrofísica y Tecnologías Afines (CATA) PFB-06/2007

\section{REFERENCES}

Arata S., Yajima H., Nagamine K., Li Y., Khochfar S., 2019, MNRAS, 488, 2629

Arata S., Yajima H., Nagamine K., Abe M., Khochfar S., 2020, arXiv e-prints, p. arXiv:2001.01853

Asplund M., Grevesse N., Sauval A. J., Scott P., 2009, ARA\&A, 47,481

Aubert D., Teyssier R., 2008, MNRAS, 387, 295

Behroozi P. S., Wechsler R. H., Wu H.-Y., 2013, ApJ, 762, 109

Behroozi P., Wechsler R. H., Hearin A. P., Conroy C., 2019, MNRAS, 488, 3143

Bigiel F., Leroy A., Walter F., Blitz L., Brinks E., de Blok W. J. G., Madore B., 2010, AJ, 140, 1194

Bovino S., Grassi T., Capelo P. R., Schleicher D. R. G., Banerjee R., 2016, A\&A, 590, A15

Brisbin D., Ferkinhoff C., Nikola T., Parshley S., Stacey G. J., Spoon H., Hailey-Dunsheath S., Verma A., 2015, ApJ, 799, 13

Bruzual G., Charlot S., 2003, MNRAS, 344, 1000

Capelo P. R., Bovino S., Lupi A., Schleicher D. R. G., Grassi T., 2018, MNRAS, 475, 3283

Carniani S., et al., 2017, A\&A, 605, A105

Carniani S., et al., 2018, MNRAS, 478, 1170

Da Cunha E., et al., 2013, Astrophysical Journal, 766

Daddi E., et al., 2010, ApJ, 714, L118

Dayal P., Ferrara A., 2018, Phys. Rep., 780, 1

De Looze I., et al., 2014, A\&A, 568, A62

Díaz-Santos T., et al., 2017, ApJ, 846, 32

Egami E., et al., 2018, Publ. Astron. Soc. Australia, 35, 48

Ferland G. J., Korista K. T., Verner D. A., Ferguson J. W., Kingdon J. B., Verner E. M., 1998, PASP, 110, 761

Ferland G. J., et al., 2017, Rev. Mex. Astron. Astrofis., 53, 385

Ferrara A., Vallini L., Pallottini A., Gallerani S., Carniani S., Kohandel M., Decataldo D., Behrens C., 2019, MNRAS, 489, 1

Glover S. C. O., Jappsen A. K., 2007, ApJ, 666, 1

Gnedin N. Y., Abel T., 2001, New Astron., 6, 437

Grassi T., Bovino S., Schleicher D. R. G., Prieto J., Seifried D., Simoncini E., Gianturco F. A., 2014, MNRAS, 439, 2386

Grassi T., Bovino S., Haugbølle T., Schleicher D. R. G., 2017, MNRAS, 466, 1259

Haardt F., Madau P., 2012, ApJ, 746, 125 
Hahn O., Abel T., 2013, MUSIC: MUlti-Scale Initial Conditions, Astrophysics Source Code Library (ascl:1311.011)

Harikane Y., et al., 2019, arXiv e-prints, p. arXiv:1910.10927

Hashimoto T., et al., 2019, PASJ, 71, 71

Herrera-Camus R., et al., 2015, ApJ, 800, 1

Hopkins P. F., 2015, MNRAS, 450, 53

Hopkins P. F., et al., 2018, MNRAS, 477, 1578

Hopkins P. F., Grudić M. Y., Wetzel A., Kereš D., FaucherGiguère C.-A., Ma X., Murray N., Butcher N., 2020, MNRAS, 491, 3702

Katz H., Kimm T., Sijacki D., Haehnelt M. G., 2017, MNRAS, 468,4831

Katz H., et al., 2019, MNRAS, 487, 5902

Kaufman M. J., Wolfire M. G., Hollenbach D. J., Luhman M. L., 1999, ApJ, 527, 795

Kennicutt Jr. R. C., 1998, ApJ, 498, 541

Kim J.-h., et al., 2014, ApJS, 210, 14

Knollmann S. R., Knebe A., 2009, ApJS, 182, 608

Kohandel M., Pallottini A., Ferrara A., Zanella A., Behrens C., Carniani S., Gallerani S., Vallini L., 2019, MNRAS, 487, 3007

Kroupa P., 2001, MNRAS, 322, 231

Levermore C., 1984, Journal of Quantitative Spectroscopy and Radiative Transfer, 31, 149

Lupi A., 2019, MNRAS, 484, 1687

Lupi A., Bovino S., 2020, MNRAS, 492, 2818

Lupi A., Bovino S., Capelo P. R., Volonteri M., Silk J., 2018, MNRAS, 474, 2884

Lupi A., Volonteri M., Decarli R., Bovino S., Silk J., Bergeron J., 2019, MNRAS, 488, 4004

Ma X., et al., 2018, MNRAS, 478, 1694

Maio U., Tescari E., 2015, MNRAS, 453, 3798

Marrone D. P., et al., 2018, Nature, 553, 51

Martizzi D., Faucher-Giguère C.-A., Quataert E., 2015, MNRAS, 450,504

Miettinen O., Delvecchio I., Smolčić V., Aravena M., Brisbin D., Karim A., 2017, A\&A, 602, L9

Olsen K. P., Greve T. R., Narayanan D., Thompson R., Toft S., Brinch C., 2015, ApJ, 814, 76

Olsen K., Greve T. R., Narayanan D., Thompson R., Davé R., Niebla Rios L., Stawinski S., 2017, ApJ, 846, 105

Olsen K., et al., 2018, Galaxies, 6, 100

Oppenheimer B. D., Schaye J., 2013, MNRAS, 434, 1043

Padoan P., Haugbølle T., Nordlund A., 2012, ApJ, 759, L27

Pallottini A., Ferrara A., Gallerani S., Vallini L., Maiolino R., Salvadori S., 2017a, MNRAS, 465, 2540

Pallottini A., Ferrara A., Bovino S., Vallini L., Gallerani S., Maiolino R., Salvadori S., 2017b, MNRAS, 471, 4128

Pallottini A., et al., 2019, MNRAS, 487, 1689

Pavesi R., Riechers D. A., Faisst A. L., Stacey G. J., Capak P. L., 2019, ApJ, 882, 168

Planck Collaboration et al., 2016, A\&A, 594, A13

Popping G., Behroozi P. S., Peeples M. S., 2015, Monthly Notices of the Royal Astronomical Society, 449, 477

Richings A. J., Schaye J., 2016, MNRAS, 458, 270

Richings A. J., Schaye J., Oppenheimer B. D., 2014, MNRAS, 442,2780

Rosdahl J., Blaizot J., Aubert D., Stranex T., Teyssier R., 2013, MNRAS, 436, 2188

Rybak M., Zavala J. A., Hodge J. A., Casey C. M., Werf P. v. d., 2020, ApJ, 889, L11

Safranek-Shrader C., Krumholz M. R., Kim C.-G., Ostriker E. C., Klein R. I., Li S., McKee C. F., Stone J. M., 2017, MNRAS, 465,885

Salim S., et al., 2007, ApJS, 173, 267

Salmon B., et al., 2015, ApJ, 799, 183

Schmidt M., 1959, ApJ, 129, 243

Semenov V. A., Kravtsov A. V., Gnedin N. Y., 2017, ApJ, 845, 133
Skinner M. A., Ostriker E. C., 2013, ApJS, 206, 21

Springel V., 2005, MNRAS, 364, 1105

Trebitsch M., Volonteri M., Dubois Y., Madau P., 2018, MNRAS, 478,5607

Trebitsch M., Volonteri M., Dubois Y., 2019, MNRAS, 487, 819

Vallini L., Gallerani S., Ferrara A., Baek S., 2013, MNRAS, 433, 1567

Vallini L., Gallerani S., Ferrara A., Pallottini A., Yue B., 2015, ApJ, 813, 36

Vallini L., Ferrara A., Pallottini A., Gallerani S., 2017, MNRAS, 467,1300

Vallini L., Pallottini A., Ferrara A., Gallerani S., Sobacchi E., Behrens C., 2018, MNRAS, 473, 271

Vallini L., Ferrara A., Pallottini A., Carniani S., Gallerani S., 2020, MNRAS

Walter F., et al., 2018, ApJ, 869, L22

Zhang Z.-Y., et al., 2018, MNRAS, 481, 59

\section{APPENDIX A: THE CMB ATTENUATION}

At high redshift, CMB represents a strong diffuse background at the same frequency of the FIR lines, over which the signal is detected as contrast (Da Cunha et al. 2013; Vallini et al. 2015). As detailed in the main text, in our analysis we assume that the state populations can be affected by CMB photons. However, recent results have suggested that this effect could be much smaller than expected, except for very-low density gas where emission would be low anyway (Pallottini et al. 2017a; Arata et al. 2020). Here, we test this idea by comparing the line flux obtained by KROME with and without including the CMB effect. In Fig. A1, we show the evolution of the FIR lines emission as a function of redshift, with (thick lines) and without (thin lines) the CMB effect. In the top panel, we show the total luminosity for $[\mathrm{CII}]_{158 \mu}$ (solid lines), $[\mathrm{OI}]_{63 \mu}$ (dashed lines), and $[\mathrm{OI}]_{146 \mu}$ (dotted lines), whereas in the bottom one we show the relative difference for each line. $[\mathrm{CII}]_{158 \mu}$ is mildly affected, and the CMB impact increases with increasing redshift, up to 50 per cent at extremely high redshift. For [OI] lines, instead, CMB has negligible effect at all redshifts.

This paper has been typeset from a $\mathrm{T}_{\mathrm{E}} \mathrm{X} / \mathrm{LAT}_{\mathrm{E}} \mathrm{X}$ file prepared by the author. 


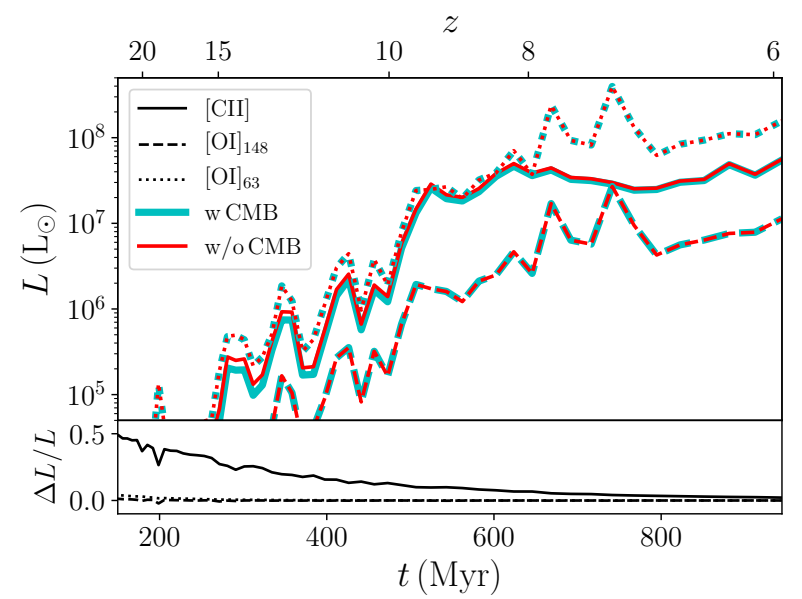

Figure A1. Cumulative line emission as a function of redshift for our galaxy, with and without including the CMB effect. In the top panel, thick/thin lines correspond to the cases with/without the $\mathrm{CMB}$, and solid, dashed and dotted styles correspond to $[\mathrm{CII}]_{158 \mu},[\mathrm{OI}]_{146 \mu}$, and $[\mathrm{OI}]_{63 \mu}$, respectively. In the bottom panel, we show the relative difference due to the CMB. We can clearly see that the effect becomes large for $[\mathrm{CII}]_{158 \mu}$ only at very high redshift, and it does not exceed 10 per cent between $z=6$ and $z=9$. [OI] lines, instead, are not affected at all by the CMB in the typical conditions we find in our simulation, but the results could significantly change with the gas temperature, since [OI] emission is more strongly influenced by temperature variations than $[\mathrm{CII}]_{158 \mu}$. 